\title{
Hyperbolicity of Direct Products of Graphs
}

\author{
Walter Carballosa ${ }^{1,2}$, Amauris de la Cruz ${ }^{3}$, Alvaro Martínez-Pérez ${ }^{4}$ (i) \\ and José M. Rodríguez ${ }^{3, * \text { iD }}$ \\ 1 Department of Mathematics and Statistics, Florida International University, 11200 SW 8th Street, \\ Miami, FL 33199, USA; waltercarb@gmail.com \\ 2 Department of Mathematics, Miami Dade College, 300 NE Second Ave. Miami, FL 33132, USA \\ 3 Departamento de Matemáticas, Universidad Carlos III de Madrid, Avenida de la Universidad 30, \\ Leganés, 28911 Madrid, Spain; alcruz@math.uc3m.es \\ 4 Facultad CC. Sociales de Talavera, Universidad de Castilla La Mancha, Avda. Real Fábrica de Seda, s.n. \\ Talavera de la Reina, 45600 Toledo, Spain; alvaro.martinezperez@uclm.es \\ * Correspondence: jomaro@math.uc3m.es; Tel.: +34-91-624-9098
}

Received: 11 June 2018; Accepted: 9 July 2018; 12 July 2018

\begin{abstract}
It is well-known that the different products of graphs are some of the more symmetric classes of graphs. Since we are interested in hyperbolicity, it is interesting to study this property in products of graphs. Some previous works characterize the hyperbolicity of several types of product graphs (Cartesian, strong, join, corona and lexicographic products). However, the problem with the direct product is more complicated. The symmetry of this product allows us to prove that, if the direct product $G_{1} \times G_{2}$ is hyperbolic, then one factor is bounded and the other one is hyperbolic. Besides, we prove that this necessary condition is also sufficient in many cases. In other cases, we find (not so simple) characterizations of hyperbolic direct products. Furthermore, we obtain good bounds, and even formulas in many cases, for the hyperbolicity constant of the direct product of some important graphs (as products of path, cycle and even general bipartite graphs).
\end{abstract}

Keywords: direct product of graphs; geodesics; Gromov hyperbolicity; bipartite graphs

\section{Introduction}

An interesting topic in graph theory is the study of the different types of products of graphs [1]. In particular, given two graphs $G_{1}, G_{2}$, the direct product $G_{1} \times G_{2}$ is defined as the graph with vertices the (Cartesian) product of $V\left(G_{1}\right)$ and $V\left(G_{2}\right)$, and two vertices $\left(u_{1}, v_{1}\right),\left(u_{2}, v_{2}\right) \in V\left(G_{1} \times G_{2}\right)$ are connected by an edge if and only if $\left[u_{1}, u_{2}\right] \in E\left(G_{1}\right)$ and $\left[v_{1}, v_{2}\right] \in E\left(G_{2}\right)$. The direct product is associative and commutative. Direct product was introduced in Principia Mathematica by Russell and Whitehead.

Weichsel observed that $G_{1} \times G_{2}$ is connected if and only if the graphs $G_{1}$ and $G_{2}$ are connected and $G_{1}$ or $G_{2}$ is not a bipartite graph [2], i.e., there exists an odd cycle. The direct product is known with different names: tensor product, conjunction, categorical product, Kronecker product and cardinal product. There are many works studying several properties of direct products. These works include structural results [3-8], hamiltonian properties [9,10], and above all the well-known Hedetniemi's conjecture (see [11,12]). Imrich has an algorithm in [13] which can recognize in polynomial time if a graph is a direct product; furthermore, the algorithm provides a factorization if the graph is a direct product. This fact facilitates the computational use of the direct product of graphs.

Hyperbolic spaces are an important tool in geometry and group theory [14-16]. Gromov hyperbolicity is a meeting point for different spaces: some of them continuous (hyperbolic plane and many Riemannian manifolds with negative curvature) and some of them discrete (trees and many graphs) [14-16]. 
Gromov hyperbolicity was introduced in the context of finitely generated groups [16], and it was applied, in the science of computation, to the study of automatic groups [17,18]. Gromov hyperbolicity is useful in networking, algorithms and discrete mathematics [19-24]; also, many real networks are hyperbolic [25-29]. Besides, there are several important applications of hyperbolic spaces to the Internet [30-34] and to random graphs [35-37]. It has recently been pointed out that also some aspects of biological systems require hyperbolicity for proper functioning [38]. In [39], it was proven that, for a large class of Riemannian surfaces endowed with a metric of negative curvature, there is a very simple graph related with the surface such that the surface is hyperbolic if and only if the graph is hyperbolic; therefore, it is interesting to study hyperbolic graphs to understand hyperbolic surfaces.

All these facts show the increasing interest of hyperbolic graphs (see, e.g., $[19,24-27,32,33,35-37,39-47]$ and the references therein).

In this paper, let us denote by $G=(V, E)=(V(G), E(G))$ a connected graph with $V(G) \neq \varnothing$. We consider that the length of each edge is 1 . In addition, we assume that the graph does not have either multiple edges or loops.

Trees are the graphs with hyperbolicity constant zero. Thus, we can view the hyperbolicity constant as a measure of how "tree-like" the space is. This is an important subject (see, e.g., [48,49]).

From a computational viewpoint, we can obtain $\delta(G)$ in time $O\left(n^{3.69}\right)$ for graphs with $n$ vertices [50]. In addition, there is an algorithm which decides if a Cayley graph is hyperbolic [51]. In [52], this algorithm is improved, allowing to obtain $\delta(G)$ in time $O\left(n^{2}\right)$, but only if the graph is given in terms of its distance-matrix. However, it is usually very difficult to decide if an infinite graph is hyperbolic. Therefore, it is useful to study hyperbolicity for particular classes of graphs. There are many works dealing with the hyperbolicity of different types of graphs: median graphs [53], line graphs [54-56], cubic graphs [57], complement graphs [58], regular graphs [59], chordal graphs [25,42,45,60], planar graphs [61,62], bipartite and intersection graphs [63], vertex-symmetric graphs [64], periodic graphs [65,66], expanders [34], bridged graphs [67], short graphs [68], graph minors [69], graphs with small hyperbolicity constant [70], Mycielskian graphs [71], geometric graphs [56,72], and some types of products of graphs: Cartesian product and sum [46,73], strong product [74], lexicographic product [75], and corona and join product [76].

Some of these works give results about the hyperbolicity of some unary operations in graphs:

A line graph is hyperbolic if and only if the original graph does [54-56].

For a large class of minor graphs, the minor graph is hyperbolic if and only if the original graph does [69].

Mycielskian graphs are always hyperbolic [71].

Now, we summarize the known results about the hyperbolicity of the main class of binary operations in graphs: products of graphs.

The Cartesian product is hyperbolic if and only if one factor graph is bounded and the other one is hyperbolic [46].

The same holds for the strong product [74].

The corona product $G_{1} \diamond G_{2}$ is hyperbolic if and only if the first factor $G_{1}$ is hyperbolic, and the join $G_{1} \uplus G_{2}$ is always hyperbolic [76].

The Cartesian sum $G_{1} \oplus G_{2}$ is always hyperbolic, if the factors have at least two vertices [73].

The lexicographic product graph $G_{1} \circ G_{2}$ is hyperbolic if and only if $G_{1}$ does, if the first factor has at least two vertices [75].

The goal of this paper is the characterization in many cases of the direct product of graphs which are hyperbolic. Here, the situation is more complicated than with other products of graphs. This is partly because the direct product of two bipartite graphs (i.e., graphs without odd cycles) is already disconnected and the formula for the distance in $G_{1} \times G_{2}$ is more complicated that in the case of other products of graphs. The symmetry of this product allows us to show that, if $G_{1} \times G_{2}$ is hyperbolic, then one factor is hyperbolic and the other one is bounded (see Theorem 10). Besides, we prove that this necessary condition is also sufficient in many cases. If $G_{1}$ is a hyperbolic graph and $G_{2}$ is a bounded 
graph, then we prove that $G_{1} \times G_{2}$ is hyperbolic when $G_{2}$ has some odd cycle (Theorem 3) or $G_{1}$ and $G_{2}$ do not have odd cycles (Theorem 4). One could think that otherwise (if $G_{1}$ has some odd cycle and $G_{2}$ does not have odd cycles) this necessary condition is also sufficient; however, Theorem 15 allows constructing in an easy way examples $G_{1}, G_{2}$ (with $G_{1}$ hyperbolic and $G_{2}$ bounded) such that $G_{1} \times G_{2}$ is not hyperbolic. This shows that the characterization of hyperbolic direct products is a more difficult task when $G_{1}$ has some odd cycle and $G_{2}$ does not have odd cycles. Theorems 11 and 12 provide sufficient conditions for non-hyperbolicity and hyperbolicity, respectively. Besides, Theorems 15 and Corollary 5 characterize the hyperbolicity of $G_{1} \times G_{2}$ under some additional conditions. Furthermore, we obtain good bounds, and even formulas in many cases, for the hyperbolicity constant of the direct product of some important graphs; in particular, Theorem 18 provides the hyperbolicity constant of many direct products of bipartite graphs, and Theorems 17 and 19 give the hyperbolicity constant of many direct products of path and cycle graphs.

We want to remark that, in a general context, the hypothesis on the existence (or non-existence) of odd cycles is artificial in the context of Gromov hyperbolicity. However, it is an essential hypothesis in the works on direct products (see Theorem 1). Throughout the development of this work, we have verified that the existence of odd cycles is also essential in the study of hyperbolic product graphs.

\section{Definitions and Background}

Let $(X, d)$ be a metric space, and denote by $L$ the length associated to the distance $d$. A geodesic is a curve $g:[a, b] \rightarrow X$ satisfying $L\left(\left.g\right|_{[t, s]}\right)=d(g(t), g(s))=|t-s|$ for every $s, t \in[a, b]$ (here, $\left.g\right|_{[t, s]}$ is the restriction of $g$ to $[t, s])$. We say that the metric space $X$ is a geodesic metric space if for each $p, q \in X$ there is a geodesic connecting them; we denote by $[p q]$ any geodesic form $p$ to $q$. Hence, a geodesic metric space is a connected space. When $X$ is a graph and $p, q \in V(X),[p, q]$ denotes the edge connecting $p$ and $q$ if they are adjacent.

Along this paper, we consider the graphs as geodesic metric spaces. To do that, we identify any edge $[p, q] \in E(G)$ with the real interval $[0,1]$; therefore, the points in a graph are the vertices and also the points in the interior of the edges. Hence, we can define a natural distance on the points of a connected graph $G$ by taking shortest paths in $G$, and so, we consider $G$ as a metric graph. If $p$ and $q$ are points in different connected components of the graph, we define $d(p, q)=\infty$.

Some authors do not consider the internal points of edges in the study. Although this approach has some advantages, we prefer to consider the internal points since these graphs are geodesic metric spaces. We use this approach since to work with geodesic metric spaces provides an interesting geometric viewpoint (for instance, Theorem 2 holds for geodesic metric spaces).

Given a geodesic metric space $X$ and three points $x_{1}, x_{2}, x_{3} \in X$, the geodesic triangle $T=$ $\left\{x_{1}, x_{2}, x_{3}\right\}$ is the union of three geodesics $\left[x_{1} x_{2}\right],\left[x_{2} x_{3}\right]$ and $\left[x_{3} x_{1}\right]$. The points $x_{1}, x_{2}, x_{3}$ are the vertices of the triangle $T$. The geodesic triangle $T$ is $\delta$-thin if any side of $T$ is contained in the $\delta$-neighborhood of the union of the two other sides. We define the thin constant of the triangle $T$ by $\delta(T):=\inf \{\delta \geq 0: T$ is $\delta$-thin $\}$, and the hyperbolicity constant of the space $X$ as $\delta(X):=$ $\sup \{\delta(T): T$ is a geodesic triangle in $X\}$. The space $X$ is hyperbolic if $\delta(X)<\infty$, and it is $\delta$-hyperbolic if $X$ is hyperbolic and the constant $\delta$ satisfies $\delta \geq \delta(X)$. We say that a triangle with two identical vertices is a "bigon". Of course, each bigon in a space (which is $\delta$-hyperbolic) is $\delta$-thin. If $\left\{X_{i}\right\}_{i \in I}$ are the connected components of $X$, then we can define $\delta(X):=\sup _{i \in I} \delta\left(X_{i}\right)$, and $X$ is hyperbolic if and only if $\delta(X)<\infty$.

We want to remark that in the classical references on hyperbolicity $[14,15,77]$ appear many different definitions of Gromov hyperbolicity. However, the definitions are equivalent: if $X$ is $\delta_{1}$-hyperbolic for a definition, then it is $\delta_{2}$-hyperbolic for every definition, where the constant $\delta_{2}$ can be obtained from $\delta_{1}$.

We refer to the classical book [1] for definitions and background about direct product graphs.

We need bounds for the distance between points in the direct product. We use the definition given in [1]. 
Definition 1. Let $G_{1}=\left(V\left(G_{1}\right), E\left(G_{1}\right)\right)$ and $G_{2}=\left(V\left(G_{2}\right), E\left(G_{2}\right)\right)$ be two graphs. The direct product $G_{1} \times G_{2}$ of $G_{1}$ and $G_{2}$ has $V\left(G_{1}\right) \times V\left(G_{2}\right)$ as vertex set, so that two distinct vertices $\left(u_{1}, v_{1}\right)$ and $\left(u_{2}, v_{2}\right)$ of $G_{1} \times G_{2}$ are adjacent if $\left[u_{1}, u_{2}\right] \in E\left(G_{1}\right)$ and $\left[v_{1}, v_{2}\right] \in E\left(G_{2}\right)$.

If $G_{1}$ and $G_{2}$ are isomorphic, we write $G_{1} \simeq G_{2}$. It is clear that, if $G_{1} \simeq G_{2}$, then $\delta\left(G_{1}\right)=\delta\left(G_{2}\right)$.

It is clear that the direct product of two graphs is commutative, i.e., $G_{1} \times G_{2} \simeq G_{2} \times G_{1}$. Therefore, the conclusion of every result in this paper with some "non-symmetric" hypothesis also holds if we change the roles of $G_{1}$ and $G_{2}$ (see, e.g., Theorems 3, 4, 11, 12 and 15 and Corollary 5).

Denote by $\pi_{i}$ the projection map $\pi_{i}: V\left(G_{1} \times G_{2}\right) \rightarrow V\left(G_{i}\right)$ for $i \in\{1,2\}$. In fact, this projection is well defined as a map $\pi_{i}: G_{1} \times G_{2} \rightarrow G_{i}$ for $i \in\{1,2\}$.

We need some previous results of [1]. If $u, u^{\prime} \in V(G)$, then by a $u, u^{\prime}$-walk in $G$ we mean a path joining $u$ and $u^{\prime}$ where repeating vertices is allowed.

Proposition 1. ([1], Proposition 5.7) Suppose $(u, v)$ and $\left(u^{\prime}, v^{\prime}\right)$ are vertices of the direct product $G_{1} \times G_{2}$, and $n$ is an integer for which $G_{1}$ has $a u, u^{\prime}$-walk of length $n$ and $G_{2}$ has a v, $v^{\prime}$-walk of length $n$. Then, $G_{1} \times G_{2}$ has a walk of length $n$ from $(u, v)$ to $\left(u^{\prime}, v^{\prime}\right)$. The smallest such $n$ (if it exists) equals $d_{G_{1} \times G_{2}}\left((u, v),\left(u^{\prime}, v^{\prime}\right)\right)$. If no such $n$ exists, then $d_{G_{1} \times G_{2}}\left((u, v),\left(u^{\prime}, v^{\prime}\right)\right)=\infty$.

Proposition 2. ([1], Proposition 5.8) Suppose $x$ and $y$ are vertices of $G_{1} \times G_{2}$. Then,

$$
d_{G_{1} \times G_{2}}(x, y)=\min \left\{n \in \mathbb{N} \mid \text { each factor } G_{i} \text { has a } \pi_{i}(x), \pi_{i}(y) \text {-walk of length } n \text { for } i=1,2\right\},
$$

where it is understood that $d_{G_{1} \times G_{2}}(x, y)=\infty$ if no such $n$ exists.

Definition 2. If $G$ is a connected graph, the diameter of its vertices is

$$
\operatorname{diam} V(G):=\sup \left\{d_{G}(u, v): u, v \in V(G)\right\},
$$

and the diameter of $G$ is

$$
\operatorname{diam} G:=\sup \left\{d_{G}(x, y): x, y \in G\right\} .
$$

Corollary 1. We have for every $(u, v),\left(u^{\prime}, v^{\prime}\right) \in V\left(G_{1} \times G_{2}\right)$

$$
d_{G_{1} \times G_{2}}\left((u, v),\left(u^{\prime}, v^{\prime}\right)\right) \geq \max \left\{d_{G_{1}}\left(u, u^{\prime}\right), d_{G_{2}}\left(v, v^{\prime}\right)\right\}
$$

and, consequently,

$$
\operatorname{diam} V\left(G_{1} \times G_{2}\right) \geq \max \left\{\operatorname{diam} V\left(G_{1}\right), \operatorname{diam} V\left(G_{2}\right)\right\}
$$

Furthermore, if $d_{G_{1}}\left(u, u^{\prime}\right)$ and $d_{G_{2}}\left(v, v^{\prime}\right)$ have the same parity, then

$$
d_{G_{1} \times G_{2}}\left((u, v),\left(u^{\prime}, v^{\prime}\right)\right)=\max \left\{d_{G_{1}}\left(u, u^{\prime}\right), d_{G_{2}}\left(v, v^{\prime}\right)\right\}
$$

and, consequently,

$$
\operatorname{diam} V\left(G_{1} \times G_{2}\right)=\max \left\{\operatorname{diam} V\left(G_{1}\right), \operatorname{diam} V\left(G_{2}\right)\right\}
$$

By trivial graph, we mean a graph which has only a vertex.

The following result characterizes when a direct product is connected. By cycle, we mean a simple closed curve, i.e., a path with different vertices, unless the last one, which is equal to the first vertex. 
Theorem 1. ([1], Theorem 5.9) Suppose $G_{1}$ and $G_{2}$ are connected non-trivial graphs. If at least one of $G_{1}$ or $G_{2}$ has an odd cycle, then $G_{1} \times G_{2}$ is connected. If both $G_{1}$ and $G_{2}$ are bipartite, then $G_{1} \times G_{2}$ has exactly two connected components.

Corollary 2. ([1], Corollary 5.10) A direct product of connected non-trivial graphs is connected if and only if at most one of the factors is bipartite. In fact, the product has $2^{\max \{k, 1\}-1}$ connected components, where $k$ is the number of bipartite factors.

Consider the metric spaces $\left(X, d_{X}\right)$ and $\left(Y, d_{Y}\right)$. Given constants $\alpha \geq 1, \beta \geq 0$, a map $f: X \longrightarrow Y$ is an $(\alpha, \beta)$-quasi-isometric embedding if

$$
\alpha^{-1} d_{X}(x, y)-\beta \leq d_{Y}(f(x), f(y)) \leq \alpha d_{X}(x, y)+\beta,
$$

for $x, y \in X$. We say that $f$ is $\varepsilon$-full if for each $y \in Y$ there is $x \in X$ with $d_{Y}(f(x), y) \leq \varepsilon$.

We say that $f$ is a quasi-isometry if there exist constants $\alpha, \beta, \varepsilon$, such that $f$ is an $\varepsilon$-full $(\alpha, \beta)$-quasi-isometric embedding.

Two metric spaces $X$ and $Y$ are quasi-isometric if there exists a quasi-isometry $f: X \longrightarrow Y$. One can check that to be quasi-isometric is an equivalence relation. An $(\alpha, \beta)$-quasi-geodesic in $X$ is an $(\alpha, \beta)$-quasi-isometric embedding between an interval of $\mathbb{R}$ and $X$.

We need the following result ([15], p. 88).

Theorem 2 (Invariance of hyperbolicity). Let $f: X \longrightarrow Y$ be an $(\alpha, \beta)$-quasi-isometric embedding between the geodesic metric spaces $X$ and $Y$. If $Y$ is $\delta_{Y}$-hyperbolic, then $X$ is $\delta_{X}$-hyperbolic, where $\delta_{X}$ is a constant which just depends on $\alpha, \beta, \delta_{Y}$.

Besides, if $f$ is $\varepsilon$-full for some $\varepsilon \geq 0$ (a quasi-isometry) and $X$ is $\delta_{X}$-hyperbolic, then $Y$ is $\delta_{Y}$-hyperbolic, where $\delta_{Y}$ is a constant which just depends on $\alpha, \beta, \delta_{X}, \varepsilon$.

There are several explicit expressions for $\delta_{X}=\delta_{X}\left(\alpha, \beta, \delta_{Y}\right)$, some of them very complicated. In [78] appears the best possible formula for $\delta_{X}$ :

$$
\delta_{X}\left(\alpha, \beta, \delta_{Y}\right)=8 \alpha\left(2 \alpha^{2}\left(A_{1} b+A_{2} \delta_{Y}\right)+4 \delta_{Y}+\beta\right)
$$

for some explicit constants $A_{1}, A_{2}$.

\section{Hyperbolic Direct Products}

Let us start with a necessary condition for hyperbolicity.

Proposition 3. Let $G_{1}$ and $G_{2}$ be two unbounded connected graphs. Then, $G_{1} \times G_{2}$ is not hyperbolic.

Proof. Since $G_{1}$ and $G_{2}$ are unbounded graphs, for each positive integer $n$ there exist two geodesic paths $P_{1}:=\left[w_{1}, w_{2}\right] \cup\left[w_{2}, w_{3}\right] \cup \cdots \cup\left[w_{n-1}, w_{n}\right]$ in $G_{1}$ and $P_{2}:=\left[v_{1}, v_{2}\right] \cup\left[v_{2}, v_{3}\right] \cup \cdots \cup\left[v_{n-1}, v_{n}\right]$ in $G_{2}$. If $n$ is odd, then we can consider the geodesic triangle $T$ in $G_{1} \times G_{2}$ (see Figure 1 ) defined by the following geodesics:

$$
\begin{aligned}
& \gamma_{1}:=\left[\left(w_{1}, v_{2}\right),\left(w_{2}, v_{1}\right)\right] \cup\left[\left(w_{2}, v_{1}\right),\left(w_{3}, v_{2}\right)\right] \cup\left[\left(w_{3}, v_{2}\right),\left(w_{4}, v_{1}\right)\right] \cup \cdots \cup\left[\left(w_{n-1}, v_{1}\right),\left(w_{n}, v_{2}\right)\right], \\
& \gamma_{2}:=\left[\left(w_{1}, v_{2}\right),\left(w_{2}, v_{3}\right)\right] \cup\left[\left(w_{2}, v_{3}\right),\left(w_{1}, v_{4}\right)\right] \cup\left[\left(w_{1}, v_{4}\right),\left(w_{2}, v_{5}\right)\right] \cup \cdots \cup\left[\left(w_{1}, v_{n-1}\right),\left(w_{2}, v_{n}\right)\right], \\
& \gamma_{3}:=\left[\left(w_{2}, v_{n}\right),\left(w_{3}, v_{n-1}\right)\right] \cup\left[\left(w_{3}, v_{n-1}\right),\left(w_{4}, v_{n-2}\right)\right] \cup\left[\left(w_{4}, v_{n-2}\right),\left(w_{5}, v_{n-3}\right)\right] \cup \cdots \cup\left[\left(w_{n-1}, v_{3}\right),\left(w_{n}, v_{2}\right)\right],
\end{aligned}
$$

Corollary 1 gives that $\gamma_{1}, \gamma_{2}, \gamma_{3}$ are geodesics.

Let $m:=\frac{n+1}{2}$ and consider the vertex $\left(w_{m}, v_{m+1}\right)$ in $\gamma_{3}$. For every vertex $\left(w_{i}, v_{j}\right)$ in $\gamma_{1}, j \in\{1,2\}$, we have $d_{G_{1} \times G_{2}}\left(\left(w_{m}, v_{m+1}\right),\left(w_{i}, v_{j}\right)\right) \geq d_{G_{2}}\left(v_{m+1}, v_{j}\right) \geq m+1-2=\frac{n-1}{2}$ by Corollary 1 . We have for 
every vertex $\left(w_{i}, v_{j}\right)$ in $\gamma_{2}, i \in\{1,2\}$, by Corollary $1, d_{G_{1} \times G_{2}}\left(\left(w_{m}, v_{m+1}\right),\left(w_{i}, v_{j}\right)\right) \geq d_{G_{1}}\left(w_{m}, w_{i}\right) \geq$ $m-2=\frac{n-3}{2}$. Hence, $d_{G_{1} \times G_{2}}\left(\left(w_{m}, v_{m+1}\right), \gamma_{1} \cup \gamma_{2}\right) \geq \frac{n-3}{2}$ and $\delta\left(G_{1} \times G_{2}\right) \geq \delta(T) \geq \frac{n-3}{2}$. Since $n$ is arbitrarily large, $G_{1} \times G_{2}$ is not hyperbolic.

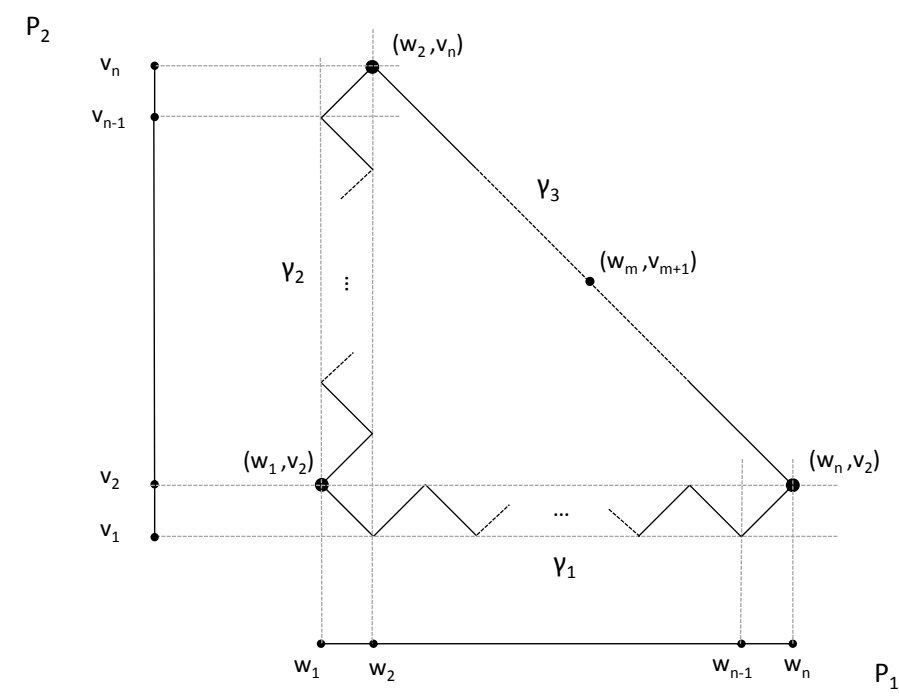

Figure 1. If $G_{1}$ and $G_{2}$ are unbounded, for any odd $n$, there is a geodesic triangle $T \subset G_{1} \times G_{2}$ with $\delta(T) \geq \frac{n-3}{2}$.

Lemma 1. Consider two connected graphs $G_{1}$ and $G_{2}$. If $f: V\left(G_{1}\right) \longrightarrow V\left(G_{2}\right)$ is an $(\alpha, \beta)$-quasi-isometric embedding, then there exists an $(\alpha, \alpha+\beta)$-quasi-isometric embedding $g: G_{1} \longrightarrow G_{2}$ with $g=f$ on $V\left(G_{1}\right)$. In addition, if $f$ is $\varepsilon-f u l l$, then $g$ is $\left(\varepsilon+\frac{1}{2}\right)$-full.

Proof. For each $x \in G_{1}$, let us choose a closest point $v_{x} \in V\left(G_{1}\right)$ from $x$, and define $g(x):=f\left(v_{x}\right)$. Note that $v_{x}=x$ if $x \in V\left(G_{1}\right)$ and so $g=f$ on $V\left(G_{1}\right)$. Given $x, y \in G_{1}$, we have

$$
\begin{aligned}
& d_{G_{2}}(g(x), g(y))=d_{G_{2}}\left(f\left(v_{x}\right), f\left(v_{y}\right)\right) \leq \alpha d_{G_{1}}\left(v_{x}, v_{y}\right)+\beta \leq \alpha\left(d_{G_{1}}(x, y)+1\right)+\beta, \\
& d_{G_{2}}(g(x), g(y))=d_{G_{2}}\left(f\left(v_{x}\right), f\left(v_{y}\right)\right) \geq \alpha^{-1} d_{G_{1}}\left(v_{x}, v_{y}\right)-\beta \geq \alpha^{-1}\left(d_{G_{1}}(x, y)-1\right)-\beta,
\end{aligned}
$$

and $g$ is an $(\alpha, \alpha+\beta)$-quasi-isometric embedding, since $\alpha \geq 1 \geq \alpha^{-1}$.

In addition, if $f$ is $\varepsilon$-full, then $g$ is $\left(\varepsilon+\frac{1}{2}\right)$-full since $g\left(G_{1}\right)=f\left(V\left(G_{1}\right)\right)$.

Given a graph $G$, let $g_{I}(G)$ denote the odd girth of $G$, that is, the length of the shortest odd cycle in $G$.

Theorem 3. Let $G_{1}$ be a connected graph and $G_{2}$ be a non-trivial bounded connected graph with some odd cycle. Then, $G_{1} \times G_{2}$ is hyperbolic if and only if $G_{1}$ is hyperbolic.

Proof. Fix $v_{0} \in V\left(G_{2}\right)$ with $v_{0}$ contained in an odd cycle $C$ with $L(C)=g_{I}\left(G_{2}\right)$. Consider the map $i: V\left(G_{1}\right) \rightarrow V\left(G_{1} \times G_{2}\right)$ such that $i(w):=\left(w, v_{0}\right)$ for every $w \in V\left(G_{1}\right)$.

By Corollary 1 , for every $w_{1}, w_{2} \in V\left(G_{1}\right), d_{G_{1}}\left(w_{1}, w_{2}\right) \leq d_{G_{1} \times G_{2}}\left(\left(w_{1}, v_{0}\right),\left(w_{2}, v_{0}\right)\right)$. In addition, Proposition 2 gives the following.

If a geodesic joining $w_{1}$ and $w_{2}$ has even length, then

$$
d_{G_{1} \times G_{2}}\left(\left(w_{1}, v_{0}\right),\left(w_{2}, v_{0}\right)\right)=d_{G_{1}}\left(w_{1}, w_{2}\right) .
$$


If a geodesic joining $w_{1}$ and $w_{2}$ has odd length, then $C$ defines a $v_{0}, v_{0}$-walk with odd length and

$$
d_{G_{1} \times G_{2}}\left(\left(w_{1}, v_{0}\right),\left(w_{2}, v_{0}\right)\right) \leq \max \left\{d_{G_{1}}\left(w_{1}, w_{2}\right), g_{I}\left(G_{2}\right)\right\} \leq d_{G_{1}}\left(w_{1}, w_{2}\right)+g_{I}\left(G_{2}\right) .
$$

Thus, $i$ is a $\left(1, g_{I}\left(G_{2}\right)\right)$ quasi-isometric embedding.

Consider any $(w, v) \in V\left(G_{1} \times G_{2}\right)$. Then, if the geodesic joining $v$ and $v_{0}$ has even length,

$$
d_{G_{1} \times G_{2}}\left((w, v),\left(w, v_{0}\right)\right)=d_{G_{2}}\left(v, v_{0}\right) .
$$

If a geodesic joining $v$ and $v_{0}$ has odd length, $\left[v v_{0}\right] \cup C$ defines a $v, v_{0}$-walk with even length. Therefore,

$$
d_{G_{1} \times G_{2}}\left((w, v),\left(w, v_{0}\right)\right) \leq d_{G_{2}}\left(v, v_{0}\right)+g_{I}\left(G_{2}\right) .
$$

Thus, $i$ is $\left(\operatorname{diam}\left(V\left(G_{2}\right)\right)+g_{I}\left(G_{2}\right)\right)$-full.

Hence, by Lemma 1 , there is a $\left(\operatorname{diam}\left(V\left(G_{2}\right)\right)+g_{I}\left(G_{2}\right)+\frac{1}{2}\right)$-full $\left(1, g_{I}\left(G_{2}\right)+1\right)$-quasi-isometry, $j: G_{1} \rightarrow G_{1} \times G_{2}$, and $G_{1} \times G_{2}$ is hyperbolic if and only if $G_{1}$ is hyperbolic by Theorem 2 .

Theorem 4. Let $G_{1}$ be a connected graph without odd cycles and $G_{2}$ be a non-trivial bounded connected graph without odd cycles. Then, $G_{1} \times G_{2}$ is hyperbolic if and only if $G_{1}$ is hyperbolic.

Proof. Fix some vertex $w_{0} \in V\left(G_{1}\right)$ and some edge $\left[v_{1}, v_{2}\right] \in E\left(G_{2}\right)$.

By Theorem 1, there are exactly two components in $G_{1} \times G_{2}$. Since there are no odd cycles, there is no $\left(w_{0}, v_{1}\right),\left(w_{0}, v_{2}\right)$-walk in $G_{1} \times G_{2}$. Thus, let us denote by $\left(G_{1} \times G_{2}\right)^{1}$ the component containing the vertex $\left(w_{0}, v_{1}\right)$ and by $\left(G_{1} \times G_{2}\right)^{2}$ the component containing the vertex $\left(w_{0}, v_{2}\right)$.

Consider $i: V\left(G_{1}\right) \rightarrow V\left(G_{1} \times G_{2}\right)^{1}$ defined as $i(w):=\left(w, v_{1}\right)$ for every $w \in V\left(G_{1}\right)$ such that every $w_{0}, w$-walk has even length and $i(w):=\left(w, v_{2}\right)$ for every $w \in V\left(G_{1}\right)$ such that every $w_{0}, w$-walk has odd length.

By Proposition 2, $d_{G_{1} \times G_{2}}\left(i\left(w_{1}\right), i\left(w_{2}\right)\right)=d_{G_{1}}\left(w_{1}, w_{2}\right)$ for every $w_{1}, w_{2} \in V\left(G_{1}\right)$ and $i$ is a (1,0)-quasi-isometric embedding.

Let $(w, v) \in V\left(G_{1} \times G_{2}\right)^{1}$. Let $v_{j}$ with $j \in\{1,2\}$ such that every $v, v_{j}$-walk has even length. Then, by Proposition $2, d_{G_{1} \times G_{2}}\left((w, v),\left(w, v_{j}\right)\right)=d_{G_{2}}\left(v, v_{j}\right) \leq \operatorname{diam}\left(G_{2}\right)$. Therefore, $i$ is $\operatorname{diam}\left(G_{2}\right)$-full.

Hence, by Lemma 1 , there is a $\left(\operatorname{diam}\left(G_{2}\right)+\frac{1}{2}\right)$-full $(1,1)$-quasi-isometry, $j: G_{1} \rightarrow\left(G_{1} \times G_{2}\right)^{1}$, and $\left(G_{1} \times G_{2}\right)^{1}$ is hyperbolic if and only if $G_{1}$ is hyperbolic by Theorem 2 .

The same argument proves that $\left(G_{1} \times G_{2}\right)^{2}$ is hyperbolic.

Denote by $P_{2}$ the path graph with two vertices and an edge.

Lemma 2. Let $G_{1}$ be a connected graph with some odd cycle and $G_{2}$ a non-trivial bounded graph without odd cycles. Then, $G_{1} \times G_{2}$ and $G_{1} \times P_{2}$ are quasi-isometric and $\delta\left(G_{1} \times P_{2}\right) \leq \delta\left(G_{1} \times G_{2}\right)$.

Proof. By Theorem 1, we know that $G_{1} \times G_{2}$ and $G_{1} \times P_{2}$ are connected graphs.

Denote by $v_{1}$ and $v_{2}$ the vertices of $P_{2}$ and fix $\left[w_{1}, w_{2}\right] \in E\left(G_{2}\right)$. The map $f: V\left(G_{1} \times P_{2}\right) \longrightarrow$ $V\left(G_{1} \times\left[w_{1}, w_{2}\right]\right)$ defined as $f\left(u, v_{j}\right):=\left(u, w_{j}\right)$ for every $u \in V\left(G_{1}\right)$ and $j=1,2$, is an isomorphism of graphs; hence, it suffices to prove that $G_{1} \times G_{2}$ and $G_{1} \times\left[w_{1}, w_{2}\right]$ are quasi-isometric.

Consider the inclusion map $i: V\left(G_{1} \times\left[w_{1}, w_{2}\right]\right) \longrightarrow V\left(G_{1} \times G_{2}\right)$. Since $G_{1} \times\left[w_{1}, w_{2}\right]$ is a subgraph of $G_{1} \times G_{2}$, we have $d_{G_{1} \times G_{2}}(x, y) \leq d_{G_{1} \times\left[w_{1}, w_{2}\right]}(x, y)$ for every $x, y \in V\left(G_{1} \times\left[w_{1}, w_{2}\right]\right)$.

Since $G_{2}$ is a graph without odd cycles, every $w_{1}, w_{2}$-walk has odd length and every $w_{j}, w_{j}$-walk has even length for $j=1,2$. Thus, Proposition 2 gives, for every $x=\left(u, w_{1}\right), y=\left(v, w_{2}\right) \in V\left(G_{1} \times\right.$ $\left.\left[w_{1}, w_{2}\right]\right)$,

$$
d_{G_{1} \times\left[w_{1}, w_{2}\right]}(x, y)=d_{G_{1} \times G_{2}}(x, y)=\min \{L(g) \mid g \text { is a } u, v \text {-walk of odd length }\} .
$$


Furthermore, for every $x=\left(u, w_{j}\right), y=\left(v, w_{j}\right) \in V\left(G_{1} \times\left[w_{1}, w_{2}\right]\right)$ and $j=1,2$,

$$
d_{G_{1} \times\left[w_{1}, w_{2}\right]}(x, y)=d_{G_{1} \times G_{2}}(x, y)=\min \{L(g) \mid g \text { is a } u, v \text {-walk of even length }\} .
$$

Hence, $d_{G_{1} \times\left[w_{1}, w_{2}\right]}(x, y)=d_{G_{1} \times G_{2}}(x, y)$ for every $x, y \in V\left(G_{1} \times\left[w_{1}, w_{2}\right]\right)$, and the inclusion map $i$ is an $(1,0)$-quasi-isometric embedding. Therefore, $\delta\left(G_{1} \times P_{2}\right)=\delta\left(G_{1} \times\left[w_{1}, w_{2}\right]\right) \leq \delta\left(G_{1} \times G_{2}\right)$.

Since $G_{2}$ is a graph without odd cycles, given any $w \in V\left(G_{2}\right)$, we have either that every $w$, $w_{1}$-walk has even length and every $w, w_{2}$-walk has odd length or that every $w, w_{2}$-walk has even length and every $w, w_{1}$-walk has odd length. In addition, since $G_{1}$ is connected, for each $u \in V\left(G_{1}\right)$ there is some $u^{\prime} \in V\left(G_{1}\right)$ such that $\left[u, u^{\prime}\right] \in E\left(G_{1}\right)$. Therefore, by Proposition 2, for every $(u, w) \in V\left(G_{1} \times G_{2}\right)$, if $\min \left\{d_{G_{2}}\left(w, w_{1}\right), d_{G_{2}}\left(w, w_{2}\right)\right\}$ is even, then

$d_{G_{1} \times G_{2}}\left((u, w), V\left(G_{1} \times\left[w_{1}, w_{2}\right]\right)\right)=d_{G_{1} \times G_{2}}\left((u, w), V\left(u \times\left[w_{1}, w_{2}\right]\right)\right)=\min \left\{d_{G_{2}}\left(w, w_{1}\right), d_{G_{2}}\left(w, w_{2}\right)\right\}$, and if $\min \left\{d_{G_{2}}\left(w, w_{1}\right), d_{G_{2}}\left(w, w_{2}\right)\right\}$ is odd, then

$d_{G_{1} \times G_{2}}\left((u, w), V\left(G_{1} \times\left[w_{1}, w_{2}\right]\right)\right)=d_{G_{1} \times G_{2}}\left((u, w), V\left(u^{\prime} \times\left[w_{1}, w_{2}\right]\right)\right)=\min \left\{d_{G_{2}}\left(w, w_{1}\right), d_{G_{2}}\left(w, w_{2}\right)\right\}$.

In both cases,

$$
d_{G_{1} \times G_{2}}\left((u, w), V\left(G_{1} \times\left[w_{1}, w_{2}\right]\right)\right) \leq \operatorname{diam} V\left(G_{2}\right),
$$

and $i$ is $\left(\operatorname{diam} V\left(G_{2}\right)\right)$-full. By Lemma 1 , there exists a $\left(\operatorname{diam} V\left(G_{2}\right)+\frac{1}{2}\right)$-full $(1,1)$-quasi-isometry $g: G_{1} \times\left[w_{1}, w_{2}\right] \longrightarrow G_{1} \times G_{2}$.

A subgraph $\Gamma$ of $G$ is said isometric if $d_{\Gamma}(x, y)=d_{G}(x, y)$ for any $x, y \in \Gamma$. One can check that $\Gamma$ is isometric if and only if $d_{\Gamma}(u, v)=d_{G}(u, v)$ for any $u, v \in V(\Gamma)$.

Lemma 3. ([47], Lemma 5) If $\Gamma$ is an isometric subgraph of $G$, then $\delta(\Gamma) \leq \delta(G)$.

A $u, v$-walk $g$ in $G$ is a shortcut of a cycle $C$ if $g \cap C=\{u, v\}$ and $L(g)<d_{C}(u, v)$ where $d_{C}$ denotes the length metric on $C$.

A cycle $C^{\prime}$ is a reduction of the cycle $C$ if both have odd length and $C^{\prime}$ is the union of a subarc $\eta$ of $C$ and a shortcut of $C$ joining the endpoints of $\eta$. Note that $L\left(C^{\prime}\right) \leq L(C)-2$. We say that a cycle is minimal if it has odd length and it does not have a reduction.

Lemma 4. If $C$ is a minimal cycle of $G$, then $L(C) \leq 4 \delta(G)$.

Proof. We prove first that $C$ is an isometric subgraph of $G$. Assume that $C$ is not an isometric subgraph. Thus, there exists a shortcut $g$ of $C$ with endpoints $u, v$. There are two subarcs $\eta_{1}, \eta_{2}$ of $C$ joining $u$ and $v$; since $C$ has odd length, we can assume that $\eta_{1}$ has even length and $\eta_{2}$ has odd length. If $g$ has even length, then $C^{\prime}:=g \cup \eta_{2}$ is a reduction of $C$. If $g$ has odd length, then $C^{\prime \prime}:=g \cup \eta_{1}$ is a reduction of $C$. Hence, $C$ is not minimal, a contradiction, and so $C$ is an isometric subgraph of $G$.

It is easy to show that any isometric cycle $C$ has length $4 \delta(C)$. This fact and Lemma 3 give $L(C)=4 \delta(C) \leq 4 \delta(G)$.

Given any $w_{0}, w_{k}$-walk $g=\left[w_{0}, w_{1}\right] \cup\left[w_{1}, w_{2}\right] \cup \cdots \cup\left[w_{k-1}, w_{k}\right]$ in $G_{1}$ and $P_{2}=\left[v_{1}, v_{2}\right]$, if $L(g)$ is either odd or even, then we define the $\left(w_{0}, v_{1}\right),\left(w_{k}, v_{i}\right)$-walk for $i \in 1,2$,

$$
\begin{aligned}
& \Gamma_{1} g:=\left[\left(w_{0}, v_{1}\right),\left(w_{1}, v_{2}\right)\right] \cup\left[\left(w_{1}, v_{2}\right),\left(w_{2}, v_{1}\right)\right] \cup\left[\left(w_{2}, v_{1}\right),\left(w_{3}, v_{2}\right)\right] \cup \cdots \cup\left[\left(w_{k-1}, v_{1}\right),\left(w_{k}, v_{2}\right)\right], \\
& \Gamma_{1} g:=\left[\left(w_{0}, v_{1}\right),\left(w_{1}, v_{2}\right)\right] \cup\left[\left(w_{1}, v_{2}\right),\left(w_{2}, v_{1}\right)\right] \cup\left[\left(w_{2}, v_{1}\right),\left(w_{3}, v_{2}\right)\right] \cup \cdots \cup\left[\left(w_{k-1}, v_{2}\right),\left(w_{k}, v_{1}\right)\right],
\end{aligned}
$$

respectively. 
Remark 1. By Proposition 2, if $g$ is a geodesic path in $G_{1}$, then $\Gamma_{1} g$ is a geodesic path in $G_{1} \times P_{2}$.

Let us define the map $R: V\left(G_{1} \times P_{2}\right) \rightarrow V\left(G_{1} \times P_{2}\right)$ as $R\left(w, v_{1}\right)=\left(w, v_{2}\right)$ and $R\left(w, v_{2}\right)=\left(w, v_{1}\right)$ for every $w \in V\left(G_{1}\right)$, and the path $\Gamma_{2} g$ as $\Gamma_{2} g=R\left(\Gamma_{1} g\right)$.

Let us define the map $\left(\Gamma_{1} g\right)^{\prime}: g \rightarrow \Gamma_{1} g$ which is an isometry on the edges and such that $\left(\Gamma_{1} g\right)^{\prime}\left(w_{j}\right)=\left(w_{j}, v_{1}\right)$ if $j$ is even and $\left(\Gamma_{1} g\right)^{\prime}\left(w_{j}\right)=\left(w_{j}, v_{2}\right)$ if $j$ is odd. In addition, let $\left(\Gamma_{2} g\right)^{\prime}: g \rightarrow \Gamma_{2} g$ be the map defined by $\left(\Gamma_{2} g\right)^{\prime}:=R \circ\left(\Gamma_{1} g\right)^{\prime}$.

Given a graph $G$, denote by $\mathcal{C}$ the set of minimal cycles of $G$.

Lemma 5. Let $G_{1}$ be a connected graph with some odd cycle and $P_{2}=\left[v_{1}, v_{2}\right]$. Consider a geodesic $g=\left[w_{0} w_{k}\right]=\left[w_{0}, w_{1}\right] \cup\left[w_{1}, w_{2}\right] \cup \cdots \cup\left[w_{k-1}, w_{k}\right]$ in $G_{1}$. Let us define $w_{0}^{\prime}:=\left(\Gamma_{1} g\right)^{\prime}\left(w_{0}\right)=\left(w_{0}, v_{1}\right)$ and $w_{k}^{\prime}:=\left(\Gamma_{2} g\right)^{\prime}\left(w_{k}\right)$, i.e., $w_{k}^{\prime}:=\left(w_{k}, v_{1}\right)$ or $w_{k}^{\prime}:=\left(w_{k}, v_{2}\right)$ if $k$ is odd or even, respectively. Then, $d_{G_{1} \times P_{2}}\left(w_{0}^{\prime}, w_{k}^{\prime}\right)>\sqrt{d_{G_{1}}\left(w_{j}, \mathcal{C}\left(G_{1}\right)\right)}$ for every $0 \leq j \leq k$.

Proof. Fix $0 \leq j \leq k$. Define

$$
\mathcal{P}:=\left\{\sigma \mid \sigma \text { is a } w_{0}, w_{k} \text {-walk such that } L(\sigma) \text { has a parity different from that of } k\right\} .
$$

Proposition 2 gives

$$
d_{G_{1} \times P_{2}}\left(w_{0}^{\prime}, w_{k}^{\prime}\right)=\min \{L(\sigma) \mid \sigma \in \mathcal{P}\} .
$$

Choose $\sigma_{0} \in \mathcal{P}$ such that $L\left(\sigma_{0}\right)=d_{G_{1} \times P_{2}}\left(w_{0}^{\prime}, w_{k}^{\prime}\right)$. Since $L(g)+L\left(\sigma_{0}\right)$ is odd, we have $L(g)+$ $L\left(\sigma_{0}\right)=2 t+1$ for some positive integer $t$. Thus, $d_{G_{1} \times P_{2}}\left(w_{0}^{\prime}, w_{k}^{\prime}\right)=L\left(\sigma_{0}\right)>\frac{1}{2}(2 t+1)$.

If $g \cup \sigma_{0}$ is a cycle, then let us define $C_{0}:=g \cup \sigma_{0}$. Thus, $L\left(C_{0}\right)=2 t+1$ and $d_{G_{1}}\left(w_{j}, C_{0}\right)=0$ for every $0 \leq j \leq k$. Otherwise, we may assume that $g \cap \sigma_{0}=\left[w_{0} w_{i_{1}}\right] \cup\left[w_{i_{2}} w_{k}\right]$ for some $0 \leq i_{1}<i_{2} \leq k$. If $\sigma_{1}=\sigma_{0} \backslash g$, then let us define $C_{0}:=\left[w_{i_{1}} w_{i_{2}}\right] \cup \sigma_{1}$ (where $\left[w_{i_{1}} w_{i_{2}}\right] \subset g$ ). Hence, $C_{0}$ is a cycle, $L\left(C_{0}\right) \leq 2 t-1$ and $d_{G_{1}}\left(w_{j}, C_{0}\right)<\frac{1}{2}(2 t+1)$.

If $C_{0}$ is not minimal, then consider a reduction $C_{1}$ of $C_{0}$. Let us repeat the process until we obtain a minimal cycle $C_{s}$. Note that $L\left(C_{1}\right) \leq L\left(C_{0}\right)-2$ and for every point $p_{1} \in C_{0}, d_{G_{1}}\left(p_{1}, C_{1}\right)<\frac{1}{2} L\left(C_{0}\right)$. Now, repeating the argument, for every $1<i \leq s, L\left(C_{i}\right) \leq L\left(C_{i-1}\right)-2$ and for every point $p_{i} \in C_{i-1}$, $d_{G_{1}}\left(p_{i}, C_{i}\right)<\frac{1}{2} L\left(C_{i-1}\right)$. Therefore,

$$
\begin{aligned}
d_{G_{1}}\left(w_{j}, \mathcal{C}\left(G_{1}\right)\right) \leq d_{G_{1}}\left(w_{j}, C_{s}\right) & \leq d_{G_{1}}\left(w_{j}, C_{0}\right)+\frac{1}{2} L\left(C_{0}\right)+\frac{1}{2} L\left(C_{1}\right)+\cdots+\frac{1}{2} L\left(C_{s}\right) \\
& <\frac{1}{2}(2 t+1)+\frac{1}{2}(2 t-1)+\cdots+\frac{5}{2}+\frac{3}{2} .
\end{aligned}
$$

Hence,

$$
d_{G_{1}}\left(w_{j}, \mathcal{C}\left(G_{1}\right)\right)<\frac{1}{2} \sum_{i=1}^{t}(2 i+1)=\frac{1}{2} t^{2}+t<\left(\frac{1}{2}(2 t+1)\right)^{2}<\left(d_{G_{1} \times P_{2}}\left(w_{0}^{\prime}, w_{k}^{\prime}\right)\right)^{2} .
$$

Corollary 3. Let $G_{1}$ be a hyperbolic connected graph with some odd cycle and $P_{2}=\left[v_{1}, v_{2}\right]$. Consider a geodesic $g=\left[w_{0} w_{k}\right]=\left[w_{0}, w_{1}\right] \cup\left[w_{1}, w_{2}\right] \cup \cdots \cup\left[w_{k-1}, w_{k}\right]$ in $G_{1}$. Let us define $w_{0}^{\prime}:=\left(\Gamma_{1} g\right)^{\prime}\left(w_{0}\right)=\left(w_{0}, v_{1}\right)$ and $w_{k}^{\prime}:=\left(\Gamma_{2} g\right)^{\prime}\left(w_{k}\right)$. Then, we have for every $0 \leq j \leq k$,

$$
\frac{1}{2}\left(k+\sqrt{d_{G_{1}}\left(w_{j}, \mathcal{C}\left(G_{1}\right)\right)}\right) \leq d_{G_{1} \times P_{2}}\left(w_{0}^{\prime}, w_{k}^{\prime}\right) \leq k+2 d_{G_{1}}\left(w_{j}, \mathcal{C}\left(G_{1}\right)\right)+4 \delta\left(G_{1}\right) .
$$

Proof. Corollary 1 and Lemma 5 give $d_{G_{1} \times P_{2}}\left(w_{0}^{\prime}, w_{k}^{\prime}\right) \geq k$ and $d_{G_{1} \times P_{2}}\left(w_{0}^{\prime}, w_{k}^{\prime}\right) \geq \sqrt{d_{G_{1}}\left(w_{j}, \mathcal{C}\left(G_{1}\right)\right)}$, and these inequalities provide the lower bound of $d_{G_{1} \times P_{2}}\left(w_{0}^{\prime}, w_{k}^{\prime}\right)$. 
Consider a geodesic $\gamma$ joining $w_{j}$ and $C \in \mathcal{C}\left(G_{1}\right)$ with $L(\gamma)=d_{G_{1}}\left(w_{j}, C\right)=d_{G_{1}}\left(w_{j}, \mathcal{C}\left(G_{1}\right)\right)$ and the $w_{0}, w_{k}$-walk

$$
g^{\prime}:=\left[w_{0} w_{j}\right] \cup \gamma \cup C \cup \gamma \cup\left[w_{j} w_{k}\right]
$$

One can check that $\Gamma_{1} g^{\prime}$ is a $w_{0}^{\prime}, w_{k}^{\prime}$-walk in $G_{1} \times P_{2}$, and so Lemma 4 gives

$$
d_{G_{1} \times P_{2}}\left(w_{0}^{\prime}, w_{k}^{\prime}\right) \leq L\left(\Gamma_{1} g^{\prime}\right)=L\left(g^{\prime}\right)=k+2 d_{G_{1}}\left(w_{j}, \mathcal{C}\left(G_{1}\right)\right)+L(C) \leq k+2 d_{G_{1}}\left(w_{j}, \mathcal{C}\left(G_{1}\right)\right)+4 \delta\left(G_{1}\right) .
$$

If $\left[v_{1}, v_{2}\right]$ is an edge of $G$, then the point $x \in\left[v_{1}, v_{2}\right]$ with $d_{G}\left(x, v_{1}\right)=d_{G}\left(x, v_{2}\right)=1 / 2$ is the midpoint of the edge $\left[v_{1}, v_{2}\right]$. Denote by $J(G)$ the set of vertices and midpoints of edges in $G$. Consider the set $\mathbb{T}_{1}(G)$ of geodesic triangles $T$ in $G$ which are cycles and such that the vertices of $T$ are in $J(G)$. We denote by $\delta_{1}(G)$ the infimum of the constants $\mu$ such that any triangle in $\mathbb{T}_{1}(G)$ is $\mu$-thin.

The following three results are used throughout the paper.

Theorem 5. ([40], Theorem 2.5) For every connected graph $G$, we have $\delta_{1}(G)=\delta(G)$.

Theorem 6. ([40], Theorem 2.6) Let $G$ be any connected graph. Then, $\delta(G)$ is always a multiple of $1 / 4$.

Theorem 7. ([40], Theorem 2.7) For any hyperbolic connected graph $G$, there exists a geodesic triangle $T \in \mathbb{T}_{1}(G)$ such that $\delta(T)=\delta(G)$.

Consider the set $\mathbb{T}_{v}(G)$ of geodesic triangles $T$ in $G$ that are cycles and such that the three vertices of the triangle $T$ are also vertices of $G . \delta_{v}(G)$ denotes the infimum of the constants $\mu$ such that every triangle in $\mathbb{T}_{v}(G)$ is $\mu$-thin.

Theorem 8. For every connected graph $G$, we have $\delta_{v}(G) \leq \delta(G) \leq 4 \delta_{v}(G)+1 / 2$. Hence, $G$ is hyperbolic if and only if $\delta_{v}(G)<\infty$. Furthermore, if $G$ is hyperbolic, then there are a geodesic triangle $T=\{a, b, c\} \in \mathbb{T}_{v}(G)$ and $q \in[a b] \cap J(G)$ such that $d(p,[a c] \cup[c b])=\delta(T)=\delta_{v}(G)$. In addition, $\delta_{v}(G)$ is an integer multiple of $1 / 2$.

Proof. The inequality $\delta_{v}(G) \leq \delta(G)$ is direct.

Consider the set $\mathbb{T}_{v}^{\prime}(G)$ of geodesic triangles $T$ in $G$ such that the three vertices of the triangle $T$ belong to $V(G)$, and denote by $\delta_{v}^{\prime}(G)$ the infimum of the constants $\mu$ such that every triangle in $\mathbb{T}_{v}^{\prime}(G)$ is $\mu$-thin. The argument in the proof of (ref. [79], Lemma 2.1) gives that $\delta_{v}^{\prime}(G)=\delta_{v}(G)$.

Let us prove now $\delta(G) \leq 4 \delta_{v}(G)+1 / 2$. Let us assume that $G$ is hyperbolic. If $\delta_{v}^{\prime}(G)=\infty$, then the inequality is trivial. Thus, it suffices to consider the case $\delta_{v}^{\prime}(G)<\infty$. By Theorem 7 , there is a triangle $T=\{a, b, c\}$ that is a cycle with $a, b, c \in J(G)$ and $q \in[a b]$ such that $d(q,[a c] \cup[c b])=\delta(T)=\delta(G)$. Assume that $a, b, c \in J(G) \backslash V(G)$ (otherwise, the argument is simpler). Let $a_{1}, a_{2}, b_{1}, b_{2}, c_{1}, c_{2} \in$ $T \cap V(G)$ such that $a \in\left[a_{1}, a_{2}\right], b \in\left[b_{1}, b_{2}\right], c \in\left[c_{1}, c_{2}\right]$ and $a_{2}, b_{1} \in[a b], c_{2}, d_{1} \in[c d], d_{2}, a_{1} \in[a c]$. Since $H:=\left\{a_{2}, b_{1}, b_{2}, c_{1}, c_{2}, a_{1}\right\}$ is a geodesic hexagon with vertices in $V(G)$, it is $4 \delta_{v}^{\prime}(G)$-thin and every point $w \in\left[b_{1}, b_{2}\right] \cup\left[b_{2} c_{1}\right] \cup\left[c_{1}, c_{2}\right] \cup\left[c_{2} a_{1}\right] \cup\left[a_{1}, a_{2}\right]$ verifies $d(w,[a c] \cup[c b]) \leq 1 / 2$, we have

$$
\begin{aligned}
\delta(G) & =d(q,[a c] \cup[c b]) \leq d\left(q,\left[b_{1}, b_{2}\right] \cup\left[b_{2} c_{1}\right] \cup\left[c_{1}, c_{2}\right] \cup\left[c_{2} a_{1}\right] \cup\left[a_{1}, a_{2}\right]\right)+1 / 2 \\
& \leq 4 \delta_{v}^{\prime}(G)+1 / 2=4 \delta_{v}(G)+1 / 2 .
\end{aligned}
$$

Assume that $G$ is not hyperbolic. Therefore, for each $M>0$ there is a triangle $T=\{a, b, c\}$ which is a cycle with $a, b, c \in J(G)$ and $q \in[a b]$ with $d(q,[a c] \cup[c b]) \geq M$. The previous argument gives $M \leq 4 \delta_{v}(G)+1 / 2$ and, since $M$ is arbitrary, we conclude $\delta_{v}(G)=\infty=\delta(G)$.

Finally, consider any geodesic triangle $T=\{a, b, c\}$ in $\mathbb{T}_{v}(G)$. Since $d(q,[a c] \cup[c b])=d(q,([a c] \cup$ $[c b]) \cap V(G)), d(q,[a c] \cup[c b])$ attains its maximum value when $q \in J(G)$. Hence, $\delta(T)$ is a multiple 
of $1 / 2$ for any triangle $T \in \mathbb{T}_{v}(G)$. Since the set of non-negative numbers that are multiple of $1 / 2$ is a discrete set, $\delta(G)$ is an integer multiple of $1 / 2$ if $G$ is hyperbolic, and there is a triangle $T=\{a, b, c\} \in \mathbb{T}_{v}(G)$ and $q \in[a b] \cap J(G)$ with $d(q,[a c] \cup[c b])=\delta(T)=\delta_{v}(G)$. This finishes the proof.

Theorem 9. If $G_{1}$ is a non-hyperbolic connected graph, then $G_{1} \times P_{2}$ is not hyperbolic.

Proof. Since $G_{1}$ is not hyperbolic, by Theorem 8 , given any $R>0$ there exists a triangle $T=\{x, y, z\}$ wich is a cycle, with $x, y, z \in V\left(G_{1}\right)$ and such that $T$ is not $R$-thin. Therefore, there exists some point $m \in T$, let us assume that $m \in[x y]$, such that $d_{G_{1}}(m,[y z] \cup[z x])>R$.

Seeking for a contradiction let us assume that $G_{1} \times P_{2}$ is $\delta$-hyperbolic.

Suppose that for some $R>\delta$, there is a geodesic triangle $T=\{x, y, z\}$ that is an even cycle in $G_{1}$, with $x, y, z \in V\left(G_{1}\right)$ and such that $T$ is not $R$-thin. Consider the (closed) path $\Lambda=[x y] \cup[y z] \cup[z x]$. Then, since $T$ has even length, the path $\Gamma_{1} \Lambda$ defines a cycle in $G_{1} \times P_{2}$. Let $\gamma_{1}, \gamma_{2}, \gamma_{3}$ be the paths in $\Gamma_{1} \Lambda$ corresponding to $[x y],[y z],[z x]$, respectively. By Corollary 1 , the curves $\gamma_{1}, \gamma_{2}$ and $\gamma_{3}$ are geodesics, and $d_{G_{1} \times P_{2}}\left(\left(\Gamma_{1} \Lambda\right)^{\prime}(m), \gamma_{2} \cup \gamma_{3}\right)>\delta$, leading to contradiction.

Suppose that, for every $R>0$, there is a geodesic triangle $T=\{x, y, z\}$ which is an odd cycle, with $x, y, z \in V\left(G_{1}\right)$ and such that $T$ is not $R$-thin.

Let $T_{1}=\{x, y, z\}$ be a geodesic triangle as above and let us assume that $\operatorname{diam}\left(T_{1}\right)=D>8 \delta$.

Let $T_{2}=\left\{x^{\prime}, y^{\prime}, z^{\prime}\right\}$ be another triangle as above such that $T_{2}$ is not $3(D+8 \delta)$-thin, this is, there is a point $m$ in one of the sides, let us call it $\sigma$, of $T_{2}$ such that $d_{G_{1}}\left(m, T_{2} \backslash \sigma\right)>3(D+8 \delta)$.

Let $g=\left[w_{0} w_{k}\right]$ with $w_{0} \in T_{1}$ and $w_{k} \in T_{2}$ be a shortest geodesic in $G_{1}$ joining $T_{1}$ and $T_{2}$ (if $T_{1}$ and $T_{2}$ intersect, just assume that $g$ is a single vertex, $w_{0}=w_{k}$, in the intersection). See Figure 2.

Let us assume that $w_{0} \in[x z]$ and $w_{k} \in\left[x^{\prime} z^{\prime}\right]$. Then, let us consider the closed path $C$ in $G_{1}$ given by the union of the geodesics in $T_{1}, g$, the geodesics in $T_{2}$ and the inverse of $g$ from $w_{k}$ to $w_{0}$, this is,

$$
C:=\left[w_{0} x\right] \cup[x y] \cup[y z] \cup\left[z w_{0}\right] \cup\left[w_{0} w_{k}\right] \cup\left[w_{k} x^{\prime}\right] \cup\left[x^{\prime} y^{\prime}\right] \cup\left[y^{\prime} z^{\prime}\right] \cup\left[z^{\prime} w_{k}\right] \cup\left[w_{k} w_{0}\right] .
$$

Since $T_{1}, T_{2}$ are odd cycles, $C$ is an even closed cycle. Therefore, $\Gamma_{1} C$ defines a cycle in $G_{1} \times P_{2}$. Moreover, by Remark $1, \Gamma_{1} C$ is a geodesic decagon in $G_{1} \times P_{2}$ with sides $\gamma_{1}=\left(\Gamma_{1} C\right)^{\prime}\left(\left[w_{0} x\right]\right), \gamma_{2}=$ $\left(\Gamma_{1} C\right)^{\prime}([x y]), \gamma_{3}=\left(\Gamma_{1} C\right)^{\prime}([y z]), \gamma_{4}=\left(\Gamma_{1} C\right)^{\prime}\left(\left[z w_{0}\right]\right), \gamma_{5}=\left(\Gamma_{1} C\right)^{\prime}\left(\left[w_{0} w_{k}\right]\right), \gamma_{6}=\left(\Gamma_{1} C\right)^{\prime}\left(\left[w_{k} x^{\prime}\right]\right)$, $\gamma_{7}=\left(\Gamma_{1} C\right)^{\prime}\left(\left[x^{\prime} y^{\prime}\right]\right), \gamma_{8}=\left(\Gamma_{1} C\right)^{\prime}\left(\left[y^{\prime} z^{\prime}\right]\right), \gamma_{9}=\left(\Gamma_{1} C\right)^{\prime}\left(\left[z^{\prime} w_{k}\right]\right)$ and $\gamma_{10}=\left(\Gamma_{1} C\right)^{\prime}\left(\left[w_{k} w_{0}\right]\right)$.

Since we are assuming that $G_{1} \times P_{2}$ is $\delta$-hyperbolic, then for every $1 \leq i \leq 10$ and every point $p \in \gamma_{i}, d_{G_{1} \times P_{2}}\left(p, C \backslash \gamma_{i}\right) \leq 8 \delta$.

Let $p:=\left(\Gamma_{1} C\right)^{\prime}(m)$.

Case 1. Suppose that $d_{G_{1}}\left(m, T_{1} \cup g\right)>8 \delta$. See Figure 2 .

By assumption, $d_{G_{1}}\left(m, T_{2} \backslash \sigma\right)>8 \delta$. If $\sigma=\left[x^{\prime} y^{\prime}\right]$ (resp. $\left.\sigma=\left[y^{\prime} z^{\prime}\right]\right)$, then $p \in \gamma_{7}$ (resp. $p \in \gamma_{8}$ ) and, by Corollary $1, d_{G_{1} \times P_{2}}\left(p, C \backslash \gamma_{7}\right)>8 \delta$ (resp. $d_{G_{1} \times P_{2}}\left(p, C \backslash \gamma_{8}\right)>8 \delta$ ) leading to contradiction. If $\sigma=\left[x^{\prime} z^{\prime}\right]$, since $\left[x^{\prime} z^{\prime}\right]=\left[x^{\prime} w_{k}\right] \cup\left[w_{k} z^{\prime}\right]$, let us assume $m \in\left[x^{\prime} w_{k}\right]$. Then, since $d_{G_{1}}\left(m, w_{k}\right)>8 \delta$, it follows that $d_{G_{1}}\left(m,\left[w_{k} z^{\prime}\right]\right)>8 \delta$. Thus, $p \in \gamma_{6}$ and, by Corollary $1, d_{G_{1} \times P_{2}}\left(p, C \backslash \gamma_{6}\right)>8 \delta$ leading to contradiction.

Case 2. Suppose that $d_{G_{1}}\left(m, T_{1} \cup g\right) \leq 8 \delta$ and $L(g) \leq 8 \delta$. See the left side of Figure 3 . Then, for every point $q$ in $T_{1} \cup g, d_{G_{1}}(m, q) \leq 8 \delta+D+8 \delta$. In particular, $d_{G_{1}}\left(m, w_{k}\right) \leq 8 \delta+D+8 \delta$. Therefore, $m \in\left[x^{\prime} z^{\prime}\right]$ and let us assume that $m \in\left[x^{\prime} w_{k}\right]$. Since $d_{G_{1}}\left(m, x^{\prime}\right) \geq d_{G_{1}}\left(m,\left[x^{\prime} y^{\prime}\right] \cup\left[y^{\prime} z^{\prime}\right]\right)>$ $3(D+8 \delta)$, there is a point $m^{\prime} \in\left[x^{\prime} m\right] \subset\left[x^{\prime} w_{k}\right]$ such that $d_{G_{1}}\left(m, m^{\prime}\right)=2(D+8 \delta)$. Then, $d_{G_{1}}\left(m^{\prime}, T_{1} \cup g\right) \geq 2(D+8 \delta)-D-8 \delta-8 \delta=D>8 \delta$. In addition, it is trivial to check that $d_{G_{1}}\left(m^{\prime},\left[x^{\prime} y^{\prime}\right] \cup\left[y^{\prime} z^{\prime}\right]\right)>3(D+8 \delta)-2(D+8 \delta)>8 \delta$ and since $\left[x^{\prime} z^{\prime}\right]$ is a geodesic, $d_{G_{1}}\left(m^{\prime},\left[z^{\prime} w_{k}\right]\right)>8 \delta$. Thus, if $p^{\prime}:=\left(\Gamma_{1} C\right)^{\prime}\left(m^{\prime}\right)$, then $p^{\prime} \in \gamma_{6}$ and, by Corollary $1, d_{G_{1} \times P_{2}}\left(p^{\prime}, C \backslash \gamma_{6}\right)>8 \delta$ leading to contradiction.

Case 3. Suppose that $d_{G_{1}}\left(m, T_{1} \cup g\right) \leq 8 \delta$ and $L(g)>8 \delta$. See the right side of Figure 3. Since $g$ is a shortest geodesic in $G_{1}$ joining $T_{1}$ and $T_{2}$, this implies that $d_{G_{1}}\left(T_{1}, T_{2}\right)>8 \delta$ and $d_{G_{1}}\left(m,\left[w_{0} w_{k}\right]\right) \leq 8 \delta$. 
Moreover, $d_{G_{1}}\left(m, w_{k}\right) \leq 16 \delta$. Otherwise, there is a point $q \in\left[w_{0} w_{k}\right]$ such that $d_{G_{1}}(m, q) \leq 8 \delta$ and $d_{G_{1}}\left(q, w_{k}\right)>8 \delta$ which means that $d_{G_{1}}\left(q, w_{0}\right)<d_{G_{1}}\left(w_{0}, w_{k}\right)-8 \delta$ and $d_{G_{1}}\left(m, w_{0}\right)<d_{G_{1}}\left(w_{0}, w_{k}\right)$ leading to contradiction.

Since $d_{G_{1}}\left(m, w_{k}\right) \leq 16 \delta, m \in\left[x^{\prime} z^{\prime}\right]$. Let us assume that $m \in\left[x^{\prime} w_{k}\right]$. Since $d_{G_{1}}\left(m,\left[x^{\prime} y^{\prime}\right] \cup\left[y^{\prime} z^{\prime}\right]\right)>$ $3(D+8 \delta)$, there is a point $m^{\prime} \in\left[x^{\prime} m\right] \subset\left[x^{\prime} w_{k}\right]$ such that $d_{G_{1}}\left(m, m^{\prime}\right)=2(D+8 \delta)$. Let us see that $d_{G_{1}}\left(m^{\prime},\left[w_{0} w_{k}\right]\right)>8 \delta$. Suppose there is some $q \in\left[w_{0} w_{k}\right]$ such that $d_{G_{1}}\left(m^{\prime}, q\right) \leq 8 \delta$. Since $m^{\prime} \in T_{2}$ and $g$ is a shortest geodesic joining $T_{1}$ and $T_{2}, d_{G_{1}}\left(q, w_{k}\right) \leq 8 \delta$. However, $32 \delta<2(D+8 \delta)=$ $d_{G_{1}}\left(m^{\prime}, m\right) \leq d_{G_{1}}\left(m^{\prime}, q\right)+d_{G_{1}}\left(q, w_{k}\right)+d_{G_{1}}\left(w_{k}, m\right) \leq 8 \delta+8 \delta+16 \delta$ which is a contradiction. Hence, $d_{G_{1}}\left(m^{\prime},\left[w_{0} w_{k}\right]\right)>8 \delta$. In addition, it is trivial to check that $d_{G_{1}}\left(m^{\prime},\left[x^{\prime} y^{\prime}\right] \cup\left[y^{\prime} z^{\prime}\right]\right)>3(D+8 \delta)-2(D+$ $8 \delta)>8 \delta$ and since $\left[x^{\prime} z^{\prime}\right]$ is a geodesic, $d_{G_{1}}\left(m^{\prime},\left[z^{\prime} w_{k}\right]\right)>8 \delta$. Thus, if $p^{\prime}:=\left(\Gamma_{1} C\right)^{\prime}\left(m^{\prime}\right)$, then $p^{\prime} \in \gamma_{6}$ and, by Corollary $1, d_{G_{1} \times P_{2}}\left(p^{\prime}, C \backslash \gamma_{6}\right)>8 \delta$ leading to contradiction.

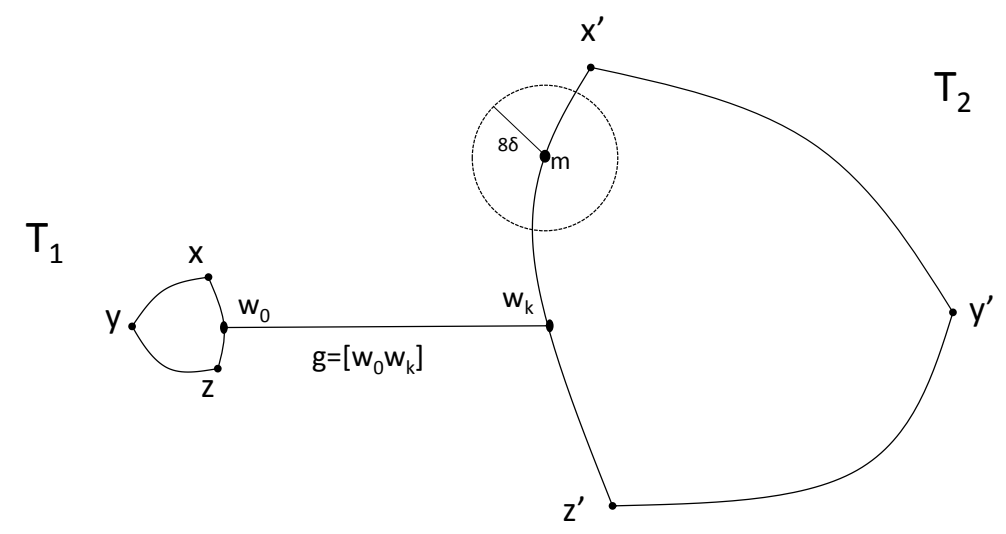

Figure 2. Two geodesic triangles, $T_{1}, T_{2}$, which are odd cycles and a geodesic $g$ joining them define an even closed path.

\section{Case 2}

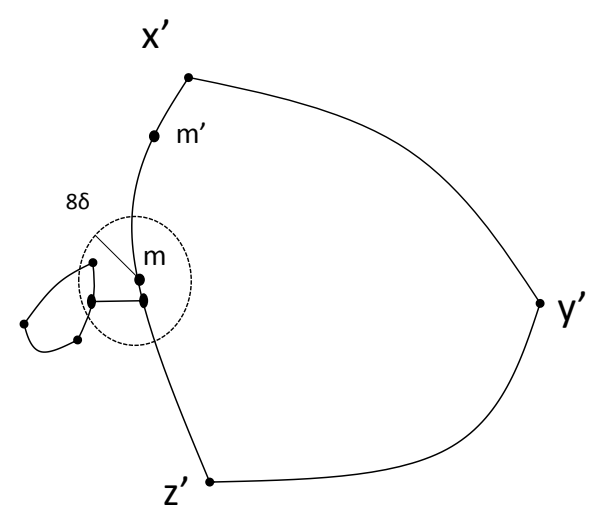

Case 3

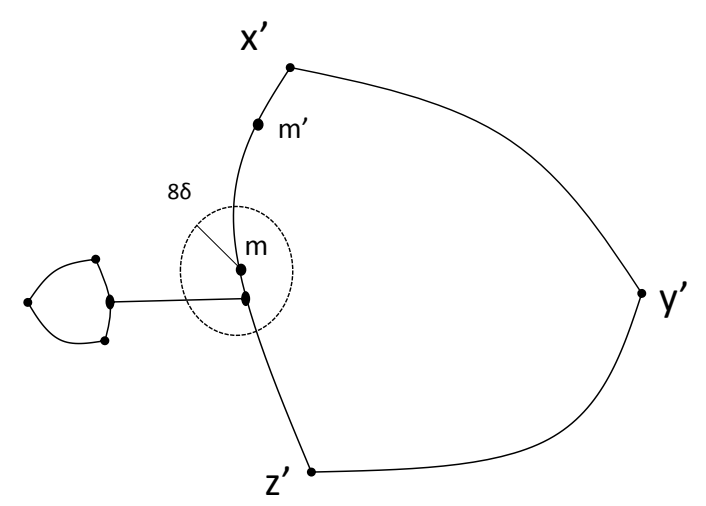

Figure 3. If $d_{G_{1}}\left(m, T_{1} \cup g\right) \leq 8 \delta$, then $m \in\left[x^{\prime} z^{\prime}\right]$ and there is a point $m^{\prime} \in\left[x^{\prime} m\right] \subset\left[x^{\prime} w_{k}\right]$ such that $d_{G_{1}}\left(m, m^{\prime}\right)=2(D+8 \delta)$.

Proposition 3, Lemma 2 and Theorems 3, 4 and 9 have the following consequence.

Corollary 4. If $G_{1}$ is a non-hyperbolic connected graph and $G_{2}$ is some non-trivial connected graph, then $G_{1} \times G_{2}$ is not hyperbolic.

Proposition 3 and Corollary 4 provide a necessary condition for the hyperbolicity of $G_{1} \times G_{2}$. 
Theorem 10. Let $G_{1}, G_{2}$ be non-trivial connected graphs. If $G_{1} \times G_{2}$ is hyperbolic, then one factor graph is hyperbolic and the other one is bounded.

Theorems 3 and 4 show that this necessary condition is also sufficient if either $G_{2}$ has some odd cycle or $G_{1}$ and $G_{2}$ do not have odd cycles (when $G_{1}$ is a hyperbolic graph and $G_{2}$ is a bounded graph). We deal now with the other case, when $G_{1}$ has some odd cycle and $G_{2}$ does not have odd cycles.

Theorem 11. Let $G_{1}$ be a connected graph with some odd cycle and $G_{2}$ a non-trivial bounded connected graph without odd cycles. Assume that $G_{1}$ satisfies the following property: for each $M>0$ there exist a geodesic $g$ joining two minimal cycles of $G_{1}$ and a vertex $u \in g \cap V\left(G_{1}\right)$ with $d_{G_{1}}\left(u, \mathcal{C}\left(G_{1}\right)\right) \geq M$. Then, $G_{1} \times G_{2}$ is not hyperbolic.

Proof. If $G_{1}$ is not hyperbolic, then Corollary 4 gives that $G_{1} \times G_{2}$ is not hyperbolic. Assume now that $G_{1}$ is hyperbolic. By Theorem 2 and Lemma 2, we can assume that $G_{2}=P_{2}$ and $V\left(P_{2}\right)=\left\{v_{1}, v_{2}\right\}$.

Fix $M>0$ and choose a geodesic $g=\left[w_{0} w_{k}\right]=\left[w_{0}, w_{1}\right] \cup\left[w_{1}, w_{2}\right] \cup \cdots \cup\left[w_{k-1}, w_{k}\right]$ joining two minimal cycles in $G_{1}$ and $0<r<k$ with $d_{G_{1}}\left(w_{r}, \mathcal{C}\left(G_{1}\right)\right) \geq M$.

Define the paths $g_{1}$ and $g_{2}$ in $G_{1} \times P_{2}$ as $g_{1}:=\Gamma_{1} g$ and $g_{2}:=\Gamma_{2} g$. Since $L\left(g_{1}\right)=L\left(g_{2}\right)=L(g)=$ $d_{G_{1}}\left(w_{0}, w_{k}\right)$, we have

$$
d_{G_{1} \times P_{2}}\left(g_{1}\left(w_{0}\right), g_{1}\left(w_{k}\right)\right) \leq L\left(g_{1}\right)=d_{G_{1}}\left(w_{0}, w_{k}\right), \quad d_{G_{1} \times P_{2}}\left(g_{2}\left(w_{0}\right), g_{2}\left(w_{k}\right)\right) \leq L\left(g_{2}\right)=d_{G_{1}}\left(w_{0}, w_{k}\right) .
$$

Corollary 1 gives that

$$
d_{G_{1} \times P_{2}}\left(g_{1}\left(w_{0}\right), g_{1}\left(w_{k}\right)\right) \geq d_{G_{1}}\left(w_{0}, w_{k}\right), \quad d_{G_{1} \times P_{2}}\left(g_{2}\left(w_{0}\right), g_{2}\left(w_{k}\right)\right) \geq d_{G_{1}}\left(w_{0}, w_{k}\right) .
$$

Hence, $g_{1}$ and $g_{2}$ are geodesics in $G_{1} \times P_{2}$. Choose geodesics $g_{3}=\left[g_{1}\left(w_{0}\right) g_{2}\left(w_{0}\right)\right]$ and $g_{4}=$ $\left[g_{1}\left(w_{k}\right) g_{2}\left(w_{k}\right)\right]$ in $G_{1} \times P_{2}$. Since $d_{P_{2}}\left(v_{1}, v_{2}\right)=1$ is odd, Proposition 2 gives

$$
\begin{aligned}
d_{G_{1} \times P_{2}}\left(g_{1}\left(w_{0}\right), g_{2}\left(w_{0}\right)\right) & =\min \left\{L(\sigma) \mid \sigma \text { is a } w_{0}, w_{0} \text {-walk }\right\} \\
& =\min \left\{L(\sigma) \mid \sigma \text { cycle of odd length containing } w_{0}\right\} .
\end{aligned}
$$

Since $w_{0}$ belongs to a minimal cycle, $L\left(g_{3}\right) \leq 4 \delta\left(G_{1}\right)$ by Lemma 4 . In a similar way, we obtain $L\left(g_{4}\right) \leq 4 \delta\left(G_{1}\right)$.

Consider the geodesic quadrilateral $Q:=\left\{g_{1}, g_{2}, g_{3}, g_{4}\right\}$ in $G_{1} \times P_{2}$. Thus, $d_{G_{1} \times P_{2}}\left(g_{1}\left(w_{r}\right), g_{2} \cup\right.$ $\left.g_{3} \cup g_{4}\right) \leq 2 \delta\left(G_{1} \times P_{2}\right)$. Since $\max \left\{L\left(g_{3}\right), L\left(g_{4}\right)\right\} \leq 4 \delta\left(G_{1}\right)$, we deduce $d_{G_{1} \times P_{2}}\left(g_{1}\left(w_{r}\right), g_{2}\right) \leq 2 \delta\left(G_{1} \times\right.$ $\left.P_{2}\right)+4 \delta\left(G_{1}\right)$.

Let $0 \leq j \leq k$ with $d_{G_{1} \times P_{2}}\left(g_{1}\left(w_{r}\right), g_{2}\right)=d_{G_{1} \times P_{2}}\left(g_{1}\left(w_{r}\right), g_{2}\left(w_{j}\right)\right)$. Let us define $w_{r}^{\prime}:=g_{1}\left(w_{r}\right)$ and $w_{j}^{\prime}:=g_{2}\left(w_{j}\right)$. Thus, Lemma 5 gives

$$
\sqrt{M} \leq \sqrt{d_{G_{1}}\left(w_{r}, \mathcal{C}\left(G_{1}\right)\right)} \leq d_{G_{1} \times P_{2}}\left(w_{r}^{\prime}, w_{j}^{\prime}\right)=d_{G_{1} \times P_{2}}\left(w_{r}^{\prime}, g_{2}\right) \leq 2 \delta\left(G_{1} \times P_{2}\right)+4 \delta\left(G_{1}\right),
$$

and since $M$ is arbitrarily large, we deduce that $G_{1} \times P_{2}$ is not hyperbolic.

Lemma 6. Let $G_{1}$ be a hyperbolic connected graph and suppose there is some constant $K>0$ such that for every vertex $w \in G_{1}, d_{G_{1}}\left(w, \mathcal{C}\left(G_{1}\right)\right) \leq K$. Then, $G_{1} \times P_{2}$ is hyperbolic.

Proof. Denote by $v_{1}$ and $v_{2}$ the vertices of $P_{2}$. Let $i: V\left(G_{1}\right) \rightarrow V\left(G_{1} \times P_{2}\right)$ defined as $i(w):=\left(w, v_{1}\right)$ for every $w \in G_{1}$.

For every $x, y \in V\left(G_{1}\right)$, by Corollary $1, d_{G_{1}}(x, y) \leq d_{G_{1} \times P_{2}}(i(x), i(y))$. By Corollary 3 ,

$$
d_{G_{1} \times P_{2}}(i(x), i(y)) \leq d_{G_{1}}(x, y)+2 d_{G_{1}}\left(x, \mathcal{C}\left(G_{1}\right)\right)+4 \delta\left(G_{1}\right) \leq d_{G_{1}}(x, y)+2 K+4 \delta\left(G_{1}\right) .
$$


Therefore, $i: V\left(G_{1}\right) \rightarrow V\left(G_{1} \times P_{2}\right)$ is a $\left(1,2 K+4 \delta\left(G_{1}\right)\right)$-quasi-isometric embedding.

Notice that for every $\left(w, v_{1}\right) \in V\left(G_{1} \times P_{2}\right),\left(w, v_{1}\right)=i(w)$. In addition, for any $\left(w, v_{2}\right) \in$ $V\left(G_{1} \times P_{2}\right)$, since $G_{1}$ is connected, there is some edge $\left[w, w^{\prime}\right] \in E\left(G_{1}\right)$ and we have $\left[\left(w, v_{2}\right),\left(w^{\prime}, v_{1}\right)\right] \in$ $E\left(G_{1} \times P_{2}\right)$. Therefore, $i: V\left(G_{1}\right) \rightarrow V\left(G_{1} \times P_{2}\right)$ is 1-full.

Thus, by Lemma $1, G_{1}$ and $G_{1} \times P_{2}$ are quasi-isometric and, by Theorem $2, G_{1} \times P_{2}$ is hyperbolic.

Theorem 3 and Lemmas 2 and 6 give the following result.

Theorem 12. Let $G_{1}$ be a hyperbolic connected graph and $G_{2}$ some non-trivial bounded connected graph. If there is some constant $K>0$ such that for every vertex $w \in G_{1}, d_{G_{1}}\left(w, \mathcal{C}\left(G_{1}\right)\right) \leq K$, then $G_{1} \times G_{2}$ is hyperbolic.

We finish this section with a characterization of the hyperbolicity of $G_{1} \times G_{2}$, under an additional hypothesis. We present first some lemmas.

Let $J$ be a finite or infinite index set. Now, given a graph $G_{1}$, we define some graphs related to $G_{1}$ which will be useful in the following results. Let $B_{j}:=B_{G_{1}}\left(w_{j}, K_{j}\right)$ with $w_{j} \in V\left(G_{1}\right)$ and $K_{j} \in \mathbb{Z}^{+}$, for any $j \in J$, such that $\sup _{j} K_{j}=K<\infty, \bar{B}_{j_{1}} \cap \bar{B}_{j_{2}}=\varnothing$ if $j_{1} \neq j_{2}$, and every odd cycle $C$ in $G_{1}$ satisfies $C \cap B_{j} \neq \varnothing$ for some $j \in J$. Denote by $G_{1}^{\prime}$ the subgraph of $G_{1}$ induced by $V\left(G_{1}\right) \backslash\left(\cup_{j} B_{j}\right)$. Let $N_{j}:=$ $\partial B_{j}=\left\{w \in V\left(G_{1}\right): d_{G_{1}}\left(w, w_{j}\right)=K_{j}\right\}$. Denote by $G_{1}^{*}$ the graph with $V\left(G_{1}^{*}\right)=V\left(G_{1}^{\prime}\right) \cup\left(\cup_{j}\left\{w_{j}^{*}\right\}\right)$, where $w_{j}^{*}$ are additional vertices, and $E\left(G_{1}^{*}\right)=E\left(G_{1}^{\prime}\right) \cup\left(\cup_{j}\left\{\left[w, w_{j}^{*}\right]: w \in N_{j}\right\}\right)$. We have $G_{1}^{\prime}=G_{1} \cap G_{1}^{*}$.

Lemma 7. Let $G_{1}$ be a connected graph as above. Then, there is a quasi-isometry $g: G_{1} \rightarrow G_{1}^{*}$ such that $g\left(w_{j}\right)=w_{j}^{*}$ for every $j \in J$.

Proof. Let $f: V\left(G_{1}\right) \rightarrow V\left(G_{1}^{*}\right)$ defined as $f(u)=u$ for every $u \in V\left(G_{1}^{\prime}\right)$, and $f(u)=w_{i}^{*}$ for every $u \in V\left(B_{i}\right)$. It is clear that $f: V\left(G_{1}\right) \rightarrow V\left(G_{1}^{*}\right)$ is 0 -full.

Now, we focus on proving that $f: V\left(G_{1}\right) \rightarrow V\left(G_{1}^{*}\right)$ is a $(K, 2 K)$-quasi-isometric embedding. For every $u, v \in V\left(G_{1}\right)$, it is clear that $d_{G_{1}^{*}}(f(u), f(v)) \leq d_{G_{1}}(u, v)$.

Let us prove the other inequality. Fix $u, v \in V\left(G_{1}\right)$ and consider an oriented geodesic $\gamma$ in $G_{1}^{*}$ from $f(u)$ to $f(v)$.

Assume that $u, v \in V\left(G_{1}^{\prime}\right)$. If $L(\gamma)=d_{G_{1}}(u, v)$, then $d_{G_{1}}(u, v)=d_{G_{1}^{*}}(f(u), f(v))$. If $L(\gamma)<$ $d_{G_{1}}(u, v)$, then $\gamma$ meets some $w_{j}^{*}$. Since $\gamma$ is a compact set, it intersects only a finite number of $w_{j}^{* \prime}$, which we denote by $w_{j_{1}}^{*}, \ldots w_{j_{r}}^{*}$. Since $\gamma$ is an oriented curve from $f(u)$ to $f(v)$, we can assume that $\gamma$ meets $w_{j_{1}}^{*}, \ldots w_{j_{r}}^{*}$ in this order.

Let us define the following vertices in $\gamma$

$$
w_{i}^{1}=\left[f(u) w_{j_{i}}^{*}\right] \cap N_{j_{i}}, \quad w_{i}^{2}=\left[w_{j_{i}}^{*} f(v)\right] \cap N_{j_{i}},
$$

for every $1 \leq i \leq r$. Note that $\left[w_{i}^{2} w_{i+1}^{1}\right] \subset G_{1}^{\prime}$ for every $1 \leq i<r$ (it is possible to have $w_{i}^{2}=w_{i+1}^{1}$ ).

Since $d_{G_{1}^{*}}\left(w_{i}^{1}, w_{i}^{2}\right)=2$ and $d_{G_{1}}\left(w_{i}^{1}, w_{i}^{2}\right) \leq 2 K$, we have $d_{G_{1}^{*}}\left(w_{i}^{1}, w_{i}^{2}\right) \geq \frac{1}{K} d_{G_{1}}\left(w_{i}^{1}, w_{i}^{2}\right)$ for every $1 \leq i \leq r$. Thus,

$$
\begin{aligned}
d_{G_{1}^{*}}(f(u), f(v)) & =d_{G_{1}^{*}}\left(f(u), w_{1}^{1}\right)+\sum_{i=1}^{r} d_{G_{1}^{*}}\left(w_{i}^{1}, w_{i}^{2}\right)+\sum_{i=1}^{r-1} d_{G_{1}^{*}}\left(w_{i}^{2}, w_{i+1}^{1}\right)+d_{G_{1}^{*}}\left(w_{r}^{2}, f(v)\right) \\
& \geq d_{G_{1}}\left(u, w_{1}^{1}\right)+\frac{1}{K} \sum_{i=1}^{r} d_{G_{1}}\left(w_{i}^{1}, w_{i}^{2}\right)+\sum_{i=1}^{r-1} d_{G_{1}}\left(w_{i}^{2}, w_{i+1}^{1}\right)+d_{G_{1}}\left(w_{r}^{2}, v\right) \\
& \geq \frac{1}{K}\left(d_{G_{1}}\left(u, w_{1}^{1}\right)+\sum_{i=1}^{r} d_{G_{1}}\left(w_{i}^{1}, w_{i}^{2}\right)+\sum_{i=1}^{r-1} d_{G_{1}}\left(w_{i}^{2}, w_{i+1}^{1}\right)+d_{G_{1}}\left(w_{r}^{2}, v\right)\right) \\
& \geq \frac{1}{K} d_{G_{1}}(u, v) .
\end{aligned}
$$


Assume that $f(u)=f(v)$. Therefore, there exists $j$ with $u, v \in B_{j}$ and

$$
d_{G_{1}^{*}}(f(u), f(v))=0>d_{G_{1}}(u, v)-2 K .
$$

Assume now that $u$ and/or $v$ does not belong to $V\left(G_{1}^{\prime}\right)$ and $f(u) \neq f(v)$. Let $u_{0}, v_{0}$ be the closest vertices in $V\left(G_{1}^{\prime}\right) \cap \gamma$ to $f(u), f(v)$, respectively (it is possible to have $u_{0}=f(u)$ or $v_{0}=f(v)$ ). Since $u_{0}, v_{0} \in V\left(G_{1}^{\prime}\right), u_{0}=f\left(u_{0}\right), v_{0}=f\left(v_{0}\right)$, we have $d_{G_{1}}\left(u, u_{0}\right)<2 K$ and $d_{G_{1}}\left(v, v_{0}\right)<2 K$. Hence,

$$
\begin{aligned}
d_{G_{1}^{*}}(f(u), f(v)) & =d_{G_{1}^{*}}\left(f(u), u_{0}\right)+d_{G_{1}^{*}}\left(u_{0}, v_{0}\right)+d_{G_{1}^{*}}\left(v_{0}, f(v)\right) \\
& \geq d_{G_{1}^{*}}\left(f\left(u_{0}\right), f\left(v_{0}\right)\right) \\
& \geq \frac{1}{K} d_{G_{1}}\left(u_{0}, v_{0}\right) \\
& \geq \frac{1}{K}\left(d_{G_{1}}(u, v)-d_{G_{1}}\left(u, u_{0}\right)-d_{G_{1}}\left(v, v_{0}\right)\right) \\
& >\frac{1}{K} d_{G_{1}}(u, v)-4 .
\end{aligned}
$$

If $K \geq 2$, then $d_{G_{1}^{*}}(f(u), f(v))>\frac{1}{K} d_{G_{1}}(u, v)-2 K$. If $K=1$, then $d_{G_{1}}\left(u, u_{0}\right) \leq 1, d_{G_{1}}\left(v, v_{0}\right) \leq 1$, and $d_{G_{1}^{*}}(f(u), f(v)) \geq d_{G_{1}}(u, v)-2$.

Finally, we conclude that $f: V\left(G_{1}\right) \rightarrow V\left(G_{1}^{*}\right)$ is a $(K, 2 K)$-quasi-isometric embedding. Thus, Lemma 1 provides a quasi-isometry $g: G_{1} \rightarrow G_{1}^{*}$ with the required property.

Definition 3. Given a connected graph $G_{1}$ and some index set $J$, let $\mathcal{B}_{J}=\left\{B_{j}\right\}_{j \in J}$ be a family of balls where $B_{j}:=B_{G_{1}}\left(w_{j}, K_{j}\right)$ with $w_{j} \in V\left(G_{1}\right), K_{j} \in \mathbb{Z}^{+}$for any $j \in J$, $\sup _{j} K_{j}=K<\infty$ and $\bar{B}_{j_{1}} \cap \bar{B}_{j_{2}}=\varnothing$ if $j_{1} \neq j_{2}$. Suppose that every odd cycle $C$ in $G_{1}$ satisfies that $C \cap B_{j} \neq \varnothing$ for some $j \in J$. If there is some constant $M>0$ such that for every $j \in J$, there is an odd cycle $C_{j}$ such that $C_{j} \cap B_{j} \neq \varnothing$ with $L\left(C_{j}\right)<M$, then we say that $\mathcal{B}_{J}$ is M-regular.

Remark 2. If J is finite, then there exists $M>0$ such that $\left\{B_{j}\right\}_{j \in J}$ is $M$-regular.

Denote by $G^{*}$ the graph with $V\left(G^{*}\right)=V\left(G_{1}^{\prime} \times P_{2}\right) \cup\left(\cup_{j}\left\{w_{j}^{*}\right\}\right)$, where $G_{1}^{\prime}$ is a graph as above and $w_{j}^{*}$ are additional vertices, and $E\left(G^{*}\right)=E\left(G_{1}^{\prime} \times P_{2}\right) \cup\left(\cup_{j}\left\{\left[w, w_{j}^{*}\right]: \pi_{1}(w) \in N_{j}\right\}\right)$.

Lemma 8. Let $G_{1}$ be a connected graph as above and $P_{2}$ with $V\left(P_{2}\right)=\left\{v_{1}, v_{2}\right\}$. If $G_{1}$ is hyperbolic and $\mathcal{B}_{J}$ as above is $M$-regular, then there exists a quasi-isometry $f: G_{1} \times P_{2} \rightarrow G^{*}$ with $f\left(w_{j}, v_{i}\right)=w_{j}^{*}$ for every $j \in J$ and $i \in\{1,2\}$.

Proof. Let $F: V\left(G_{1} \times P_{2}\right) \rightarrow V\left(G^{*}\right)$ defined as $F\left(v, v_{i}\right)=\left(v, v_{i}\right)$ for every $v \in V\left(G_{1}^{\prime}\right)$, and $F\left(v, v_{i}\right)=$ $w_{j}^{*}$ for every $v \in V\left(B_{j}\right)$. It is clear that $F: V\left(G_{1} \times P_{2}\right) \rightarrow V\left(G^{*}\right)$ is 0 -full. Recall that we denote by $\pi_{1}: G_{1} \times P_{2} \rightarrow G_{1}$ the projection map. Define $\pi^{*}: G^{*} \rightarrow G_{1}$ as $\pi^{*}=\pi_{1}$ on $G_{1}^{\prime} \times P_{2}$ and $\pi^{*}(x)=w_{j}$ for every $x$ with $d_{G^{*}}\left(x, w_{j}^{*}\right)<1$ for some $j \in J$.

Now, we focus on proving that $F: V\left(G_{1} \times P_{2}\right) \rightarrow V\left(G^{*}\right)$ is a quasi-isometric embedding. For every $\left(w, v_{i}\right),\left(w^{\prime}, v_{i^{\prime}}\right) \in V\left(G_{1} \times P_{2}\right)$, one can check

$$
d_{G^{*}}\left(F\left(w, v_{i}\right), F\left(w^{\prime}, v_{i^{\prime}}\right)\right) \leq d_{G_{1} \times P_{2}}\left(\left(w, v_{i}\right),\left(w^{\prime}, v_{i^{\prime}}\right)\right) .
$$

To prove the other inequality, let us fix $\left(w, v_{i}\right),\left(w^{\prime}, v_{i^{\prime}}\right) \in V\left(G_{1}^{\prime} \times P_{2}\right)$ (the inequalities in other cases can be obtained from the one in this case, as in the proof of Lemma 7). Consider a geodesic $\gamma:=\left[F\left(w, v_{i}\right) F\left(w^{\prime}, v_{i^{\prime}}\right)\right]$ in $G^{*}$. If $L(\gamma)=d_{G_{1} \times P_{2}}\left(\left(w, v_{i}\right),\left(w^{\prime}, v_{i^{\prime}}\right)\right)$, then

$$
d_{G^{*}}\left(F\left(w, v_{i}\right), F\left(w^{\prime}, v_{i^{\prime}}\right)\right)=d_{G_{1} \times P_{2}}\left(\left(w, v_{i}\right),\left(w^{\prime}, v_{i^{\prime}}\right)\right) .
$$


If $L(\gamma)<d_{G_{1} \times P_{2}}\left(\left(w, v_{i}\right),\left(w^{\prime}, v_{i^{\prime}}\right)\right)$, then $\pi^{*}(\gamma)$ meets some $B_{j}$. Since $\gamma$ is a compact set, $\pi^{*}(\gamma)$ intersects just a finite number of $B_{j}$ 's, which we denote by $B_{j_{1}}, \ldots B_{j_{r}}$. We consider $\gamma$ as an oriented curve from $F\left(w, v_{i}\right)$ to $F\left(w^{\prime}, v_{i^{\prime}}\right)$; thus we can assume that $\pi^{*}(\gamma)$ meets $B_{j_{1}}, \ldots, B_{j_{r}}$ in this order.

Let us define the following set of vertices in $\gamma$

$$
\left\{w_{i}^{1}, w_{i}^{2}\right\}:=\gamma \cap\left(N_{j_{i}} \times P_{2}\right),
$$

for every $1 \leq i \leq r$, such that $d_{G_{1} \times P_{2}}\left(\left(w, v_{i}\right), w_{i}^{1}\right)<d_{G_{1} \times P_{2}}\left(\left(w, v_{i}\right), w_{i}^{2}\right)$. Note that $\left[w_{i}^{2} w_{i+1}^{1}\right] \subset G_{1}^{\prime} \times P_{2}$ for every $1 \leq i<r$ and $d_{G_{1} \times P_{2}}\left(w_{i}^{2}, w_{i+1}^{1}\right) \geq 1$ since $\bar{B}_{j_{i}} \cap \bar{B}_{j_{i+1}}=\varnothing$.

If $d_{G_{1}}\left(\pi\left(w_{i}^{1}\right), \pi\left(w_{i}^{2}\right)\right)=d_{G_{1} \times P_{2}}\left(w_{i}^{1}, w_{i}^{2}\right)$ for some $1 \leq i \leq r$, then $d_{G_{1} \times P_{2}}\left(w_{i}^{1}, w_{i}^{2}\right) \leq 2 K$. Since $d_{G_{1} \times P_{2}}\left(w_{i}^{2}, w_{i+1}^{1}\right) \geq 1$ for $1 \leq i<r$, we have that $d_{G_{1} \times P_{2}}\left(w_{i}^{1}, w_{i}^{2}\right) \leq 2 K d_{G_{1} \times P_{2}}\left(w_{i}^{2}, w_{i+1}^{1}\right)$ in this case.

If $d_{G_{1}}\left(\pi_{1}\left(w_{i}^{1}\right), \pi_{1}\left(w_{i}^{2}\right)\right)<d_{G_{1} \times P_{2}}\left(w_{i}^{1}, w_{i}^{2}\right)$ for some $1 \leq i \leq r$, then $d_{G_{1}}\left(\pi_{1}\left(w_{i}^{1}\right), \pi_{1}\left(w_{i}^{2}\right)\right)+$ $d_{G_{1} \times P_{2}}\left(w_{i}^{1}, w_{i}^{2}\right)$ is odd.

Since $\mathcal{B}_{J}$ is $M$-regular, consider an odd cycle $C$ with $C \cap B_{j_{i}} \neq \varnothing$ and $L(C)<M$, and let $b_{i} \in C \cap B_{j_{i}}$ and $\left[\pi_{1}\left(w_{i}^{1}\right) b_{i}\right],\left[b_{i} \pi_{1}\left(w_{i}^{2}\right)\right]$ geodesics in $G_{1}$. Thus, $\left[\pi_{1}\left(w_{i}^{1}\right) b_{i}\right] \cup\left[b_{i} \pi_{1}\left(w_{i}^{2}\right)\right]$ and $\left[\pi_{1}\left(w_{i}^{1}\right) b_{i}\right] \cup C \cup\left[b_{i} \pi_{1}\left(w_{i}^{2}\right)\right]$ have different parity which means that one of them has different parity from $\left[\pi_{1}\left(w_{i}^{1}\right) \pi_{1}\left(w_{i}^{2}\right)\right]$. Then, $d_{G_{1} \times P_{2}}\left(w_{i}^{1}, w_{i}^{2}\right) \leq L\left(\left[\pi_{1}\left(w_{i}^{1}\right) b_{i}\right] \cup C \cup\left[b_{i} \pi_{1}\left(w_{i}^{2}\right)\right]\right) \leq 4 K+M$. Since $d_{G_{1} \times P_{2}}\left(w_{i}^{2}, w_{i+1}^{1}\right) \geq 1$ for $1 \leq i<r$, we have that $d_{G_{1} \times P_{2}}\left(w_{i}^{1}, w_{i}^{2}\right) \leq(4 K+M) d_{G_{1} \times P_{2}}\left(w_{i}^{2}, w_{i+1}^{1}\right)$ in this case.

Thus, we have that $d_{G_{1} \times P_{2}}\left(w_{i}^{1}, w_{i}^{2}\right) \leq 4 K+M$ for every $1 \leq i \leq r$ and $d_{G_{1} \times P_{2}}\left(w_{i}^{1}, w_{i}^{2}\right) \leq(4 K+$ M) $d_{G_{1} \times P_{2}}\left(w_{i}^{2}, w_{i+1}^{1}\right)$ for every $1 \leq i<r$. Therefore,

$$
\begin{aligned}
& \begin{array}{l}
d_{G_{1} \times P_{2}}\left(\left(w, v_{i}\right),\left(w^{\prime}, v_{i^{\prime}}\right)\right) \leq \\
\quad d_{G_{1} \times P_{2}}\left(\left(w, v_{i}\right), w_{1}^{1}\right)+\sum_{i=1}^{r} d_{G_{1} \times P_{2}}\left(w_{i}^{1}, w_{i}^{2}\right)+\sum_{i=1}^{r-1} d_{G_{1} \times P_{2}}\left(w_{i}^{2}, w_{i+1}^{1}\right) \\
\quad+d_{G_{1} \times P_{2}}\left(w_{r^{\prime}}^{2},\left(w^{\prime}, v_{i^{\prime}}\right)\right)
\end{array} \\
& \begin{array}{c}
\leq d_{G_{1} \times P_{2}}\left(\left(w, v_{i}\right), w_{1}^{1}\right)+d_{G_{1} \times P_{2}}\left(w_{r}^{2},\left(w^{\prime}, v_{i^{\prime}}\right)\right)+(4 K+M+1) \sum_{i=1}^{r-1} d_{G_{1} \times P_{2}}\left(w_{i}^{2}, w_{i+1}^{1}\right) \\
\quad+d_{G_{1} \times P_{2}}\left(w_{r^{\prime}}^{1}, w_{r}^{2}\right)
\end{array} \\
& =d_{G^{*}}\left(F\left(w, v_{i}\right), F\left(w_{1}^{1}\right)\right)+d_{G^{*}}\left(F\left(w_{r}^{2}\right), F\left(w^{\prime}, v_{i^{\prime}}\right)\right)+(4 K+M+1) \sum_{i=1}^{r-1} d_{G^{*}}\left(F\left(w_{i}^{2}\right), F\left(w_{i+1}^{1}\right)\right) \\
& \quad+d_{G_{1} \times P_{2}}\left(w_{r^{\prime}}^{1}, w_{r}^{2}\right)
\end{aligned}
$$

We conclude that $F: V\left(G_{1} \times P_{2}\right) \rightarrow V\left(G^{*}\right)$ is a quasi-isometric embedding. Thus, Lemma 1 provides a quasi-isometry $f: G_{1} \times P_{2} \rightarrow G^{*}$ with the required property.

Definition 4. Given a geodesic metric space $X$ and closed connected pairwise disjoint subsets $\left\{\eta_{j}\right\}_{j \in J}$ of $X$, we consider another copy $X^{\prime}$ of $X$. The double $D X$ of $X$ is the union of $X$ and $X^{\prime}$ obtained by identifying the corresponding points in each $\eta_{j}$ and $\eta_{j}^{\prime}$.

Definition 5. Let us consider $H>0$, a metric space $X$, and subsets $Y, Z \subseteq X$. The set $V_{H}(Y):=\{x \in X$ : $d(x, Y) \leq H\}$ is called the $H$-neighborhood of $Y$ in $X$. The Hausdorff distance of $Y$ to $Z$ is defined by $\mathcal{H}(Y, Z):=\inf \left\{H>0: Y \subseteq V_{H}(Z), Z \subseteq V_{H}(Y)\right\}$.

The following results in $[15,80]$ will be useful. 
Theorem 13. ([80], Theorem 3.2) Let us consider a geodesic metric space $X$ and closed connected pairwise disjoint subsets $\left\{\eta_{j}\right\}_{j \in J}$ of $X$, such that the double DX is a geodesic metric space. Then, the following conditions are equivalent:

(1) DX is hyperbolic.

(2) $X$ is hyperbolic and there exists a constant $c_{1}$ such that for every $k, l \in J$ and $a \in \eta_{k}, b \in \eta_{l}$ we have $d_{X}\left(x, \cup_{j \in J} \eta_{j}\right) \leq c_{1}$ for every $x \in[a b] \subset X$.

(3) $X$ is hyperbolic and there exist constants $c_{2}, \alpha, \beta$ such that for every $k, l \in J$ and $a \in \eta_{k}, b \in \eta_{l}$ we have $d_{X}\left(x, \cup_{j \in J} \eta_{j}\right) \leq c_{2}$ for every $x$ in some $(\alpha, \beta)$-quasi-geodesic joining a with $b$ in $X$.

Theorem 14. ([15], p. 87) For each $\delta \geq 0, a \geq 1$ and $b \geq 0$, there exists a constant $H=H(\delta, a, b)$ with the following property:

Let us consider a $\delta$-hyperbolic geodesic metric space $X$ and an $(a, b)$-quasigeodesic $g$ starting in $x$ and finishing in $y$. If $\gamma$ is a geodesic joining $x$ and $y$, then $\mathcal{H}(g, \gamma) \leq H$.

This property is called geodesic stability. It is well-known that hyperbolicity is, in fact, equivalent to geodesic stability [81].

Theorem 15. Let $G_{1}$ be a connected graph and $B_{j}:=B_{G_{1}}\left(w_{j}, K_{j}\right)$ with $w_{j} \in V\left(G_{1}\right)$ and $K_{j} \in \mathbb{Z}^{+}$, for any $j \in J$, such that $\sup _{j} K_{j}=K<\infty, \bar{B}_{j_{1}} \cap \bar{B}_{j_{2}}=\varnothing$ if $j_{1} \neq j_{2}$, and every odd cycle $C$ in $G_{1}$ satisfies $C \cap B_{j} \neq \varnothing$ for some $j \in J$. Suppose $\left\{B_{j}\right\}_{j \in J}$ is M-regular for some $M>0$. Let $G_{2}$ be a non-trivial bounded connected graph without odd cycles. Then, the following statements are equivalent:

(1) $G_{1} \times G_{2}$ is hyperbolic.

(2) $G_{1}$ is hyperbolic and there exists a constant $c_{1}$, such that for every $k, l \in J$ and $w_{k} \in B_{k}, w_{l} \in B_{l}$ there exists a geodesic $\left[w_{k} w_{l}\right]$ in $G_{1}$ with $d_{G_{1}}\left(x, \cup_{j \in J} w_{j}\right) \leq c_{1}$ for every $x \in\left[w_{k} w_{l}\right]$.

(3) $G_{1}$ is hyperbolic and there exist constants $c_{2}, \alpha, \beta$, such that for every $k, l \in J$ we have $d_{G_{1}}\left(x, \cup_{j \in J} w_{j}\right) \leq c_{2}$ for every $x$ in some $(\alpha, \beta)$-quasi-geodesic joining $w_{k}$ with $w_{l}$ in $G_{1}$.

Proof. Items (2) and (3) are equivalent by geodesic stability in $G_{1}$ (see Theorem 14).

Assume that (2) holds. By Lemma 7, there exists an $(\alpha, \beta)$-quasi-isometry $f: G_{1} \rightarrow G_{1}^{*}$ with $f\left(w_{j}\right)=w_{j}^{*}$ for every $j \in J$. Given $k, l \in J, f\left(\left[w_{k} w_{l}\right]\right)$ is an $(\alpha, \beta)$-quasi-geodesic with endpoints $w_{k}^{*}$ and $w_{l}^{*}$ in $G_{1}^{*}$. Given $x \in f\left(\left[w_{k} w_{l}\right]\right)$, we have $x=f\left(x_{0}\right)$ with $x_{0} \in\left[w_{k} w_{l}\right]$ and $d_{G_{1}^{*}}\left(x, \cup_{j \in j} w_{j}^{*}\right) \leq$ $\alpha d_{G_{1}}\left(x_{0}, \cup_{j \in J} w_{j}\right)+\beta \leq \alpha c_{1}+\beta$. Taking $X=G_{1}^{*}, D X=G^{*}$ and $\eta_{j}=w_{j}^{*}$ for every $j \in J$, Theorem 13 gives that $G^{*}$ is hyperbolic. Now, Lemma 8 gives that $G_{1} \times P_{2}$ is hyperbolic and we conclude that $G_{1} \times G_{2}$ is hyperbolic by Lemma 2 .

Now, suppose (1) holds. By Lemma 2, $G_{1} \times P_{2}$ is hyperbolic and, by Theorem 9, $G_{1}$ is hyperbolic. Then, Lemma 8 gives that $G^{*}$ is hyperbolic and taking $X=G_{1}^{*}, D X=G^{*}$ and $\eta_{j}=w_{j}^{*}$ for every $j \in J$, by Theorem 13, (2) holds.

Theorem 15 and Remark 2 have the following consequence.

Corollary 5. Let $G_{1}$ be a connected graph and suppose that there are a positive integer $K$ and a vertex $w \in G_{1}$, such that every odd cycle in $G_{1}$ intersects the open ball $B:=B_{G_{1}}(w, K)$. Let $G_{2}$ be a non-trivial bounded connected graph without odd cycles. Then, $G_{1} \times G_{2}$ is hyperbolic if and only if $G_{1}$ is hyperbolic.

\section{Bounds for the Hyperbolicity Constant of Some Direct Products}

The following well-known result will be useful (see a proof, e.g., in ([47], Theorem 8)).

Theorem 16. In any connected graph $G$ the inequality $\delta(G) \leq(\operatorname{diam} G) / 2$ holds.

Remark 3. Note that, if $G_{1}$ is a bipartite connected graph, then diam $G_{1}=\operatorname{diam} V\left(G_{1}\right)$. Furthermore, if $G_{2}$ is a bipartite connected graph, then the product $G_{1} \times G_{2}$ has exactly two connected components, which are denoted 
by $\left(G_{1} \times G_{2}\right)^{1}$ and $\left(G_{1} \times G_{2}\right)^{2}$, where each one is a bipartite graph and, consequently, $\operatorname{diam}\left(G_{1} \times G_{2}\right)^{i}=$ diam $V\left(\left(G_{1} \times G_{2}\right)^{i}\right)$ for $i \in\{1,2\}$.

Remark 4. Let $P_{m}, P_{n}$ be two path graphs with $m \geq n \geq 2$. The product $P_{m} \times P_{n}$ has exactly two connected components, which will be denoted by $\left(P_{m} \times P_{n}\right)^{1}$ and $\left(P_{m} \times P_{n}\right)^{2}$. If $u, v \in V\left(\left(P_{m} \times P_{n}\right)^{i}\right)$ for $i \in\{1,2\}$, then $d_{\left(P_{m} \times P_{n}\right)^{i}}(u, v)=\max \left\{d_{P_{m}}\left(\pi_{1}(u), \pi_{1}(v)\right), d_{P_{n}}\left(\pi_{2}(u), \pi_{2}(v)\right)\right\}$ and $\operatorname{diam}\left(P_{m} \times P_{n}\right)^{i}=$ $\operatorname{diam} V\left(\left(P_{m} \times P_{n}\right)^{i}\right)=m-1$.

Furthermore, if $m_{1} \leq m$ and $n_{1} \leq n$, then $\delta\left(P_{m} \times P_{n}\right) \geq \delta\left(P_{m_{1}} \times P_{n_{1}}\right)$.

Lemma 9. Let $P_{m}, P_{n}$ be two path graphs with $m \geq n \geq 3$, and let $\gamma$ be a geodesic in $P_{m} \times P_{n}$ such that there are two different vertices $u, v$ in $\gamma$, with $\pi_{1}(u)=\pi_{1}(v)$. Then, $L(\gamma) \leq n-1$.

Remark 5. Note that the conclusion of Lemma 9 does not hold for $n=2$, since we always have $L(\gamma) \geq 2$.

Proof. Let $\gamma:=[x y]$, and let $V\left(P_{m}\right)=\left\{v_{1}, \ldots, v_{m}\right\}, V\left(P_{n}\right)=\left\{w_{1}, \ldots, w_{n}\right\}$ be the sets of vertices in $P_{m}, P_{n}$, respectively, such that $\left[v_{j}, v_{j+1}\right] \in E\left(P_{m}\right)$ and $\left[w_{i}, w_{i+1}\right] \in E\left(P_{n}\right)$ for $1 \leq j<m, 1 \leq i<n$. Seeking for a contradiction, assume that $L(\gamma)>n-1$. Notice that if $[u v]$ denotes the geodesic contained in $\gamma$ joining $u$ and $v$, then $\pi_{2}$ restricted to $[u v]$ is injective. Consider two vertices $u^{\prime}, v^{\prime} \in \gamma$ such that $[u v] \subseteq\left[u^{\prime} v^{\prime}\right] \subseteq \gamma, \pi_{2}$ is injective in $\left[u^{\prime} v^{\prime}\right]$ and $\pi_{2}\left(u^{\prime}\right)=w_{i_{1}}, \pi_{2}\left(v^{\prime}\right)=w_{i_{2}}$ with $i_{2}-i_{1}$ maximal under these conditions. See Figure 4.

Since $L(\gamma)>n-1 \geq i_{2}-i_{1}$, either there is an edge $\left[v^{\prime}, w\right]$ in $G_{1} \times G_{2}$ such that $\left[v^{\prime}, w\right] \cap(\gamma \backslash$ $\left.\left[u^{\prime} v^{\prime}\right]\right) \neq \varnothing$ or there is an edge $\left[u^{\prime}, w^{\prime}\right]$ in $G_{1} \times G_{2}$ such that $\left[u^{\prime}, w^{\prime}\right] \cap\left(\gamma \backslash\left[u^{\prime} v^{\prime}\right]\right) \neq \varnothing$. In addition, since $L(\gamma)>n-1$, notice that $\pi_{2}$ is not injective in $\gamma$. Moreover, since $i_{2}-i_{1}$ is maximal, if $\pi_{2}(w)=$ $w_{i_{2}+1}$, then $w \notin \gamma$, and since $L(\gamma)>n-1, u^{\prime} \notin\{x, y\}$ and $\pi_{2}\left(w^{\prime}\right)=w_{i_{1}+1}$. Thus, either $\pi_{2}(w)=w_{i_{2}-1}$ or $\pi_{2}\left(w^{\prime}\right)=w_{i_{1}+1}$.

Hence, let us assume that there is an edge $\left[v^{\prime}, w\right]$ in $G_{1} \times G_{2}$ such that $\left[v^{\prime}, w\right] \cap\left(\gamma \backslash\left[u^{\prime} v^{\prime}\right]\right) \neq \varnothing$ with $\pi_{2}(w)=w_{i_{2}-1}$ (otherwise, if there is an edge $\left[u^{\prime}, w^{\prime}\right]$ in $G_{1} \times G_{2}$ such that $\left[u^{\prime}, w^{\prime}\right] \cap\left(\gamma \backslash\left[u^{\prime} v^{\prime}\right]\right) \neq \varnothing$ with $\pi_{2}\left(w^{\prime}\right)=w_{i_{1}+1}$, the proof is similar).

Suppose $\pi_{1}\left(v^{\prime}\right)=v_{j}$. Let $v^{\prime \prime}$ be the vertex in $\left[u^{\prime} v^{\prime}\right]$ such that $\pi_{2}\left(v^{\prime \prime}\right)=w_{i_{2}-1}$. Then, by construction of $G_{1} \times G_{2}$, since $v^{\prime \prime} \neq w$, it follows that $\left\{\pi_{1}\left(v^{\prime \prime}\right), \pi_{1}(w)\right\}=\left\{v_{j-1}, v_{j+1}\right\}$. Therefore, in particular, $1<j<m$.

Assume that $v^{\prime \prime}=\left(v_{j-1}, w_{i_{2}-1}\right)$ (if $v^{\prime \prime}=\left(v_{j+1}, w_{i_{2}-1}\right)$, then the argument is similar). Therefore, $w=\left(v_{j+1}, w_{i_{2}-1}\right)$.

Consider the geodesic

$$
\sigma=\left[\left(v_{j+1}, w_{i_{2}-1}\right),\left(v_{j}, w_{i_{2}-2}\right)\right] \cup\left[\left(v_{j}, w_{i_{2}-2}\right),\left(v_{j-1}, w_{i_{2}-3}\right)\right] \cup\left[\left(v_{j-1}, w_{i_{2}-3}\right),\left(v_{j-2}, w_{i_{2}-4}\right)\right] \cup \ldots
$$

Since $\pi_{1}(u)=\pi_{1}(v)$, there is a vertex $\xi$ of $V\left(P_{m} \times P_{n}\right)$ in $\left[u^{\prime} v^{\prime}\right] \cap \sigma$. Let $s \in\left[v^{\prime}, w\right] \cap \gamma$ with $s \neq v^{\prime}$. Let $\sigma_{0}$ be the geodesic contained in $\sigma$ joining $\xi$ and $w$. Let $\gamma_{0}$ be the geodesic contained in $\gamma$ joining $\xi$ and $s$. Hence, $L\left(\sigma_{0} \cup[w s]\right)<L\left(\sigma_{0}\right)+1<L\left(\gamma_{0}\right)$ leading to contradiction. 


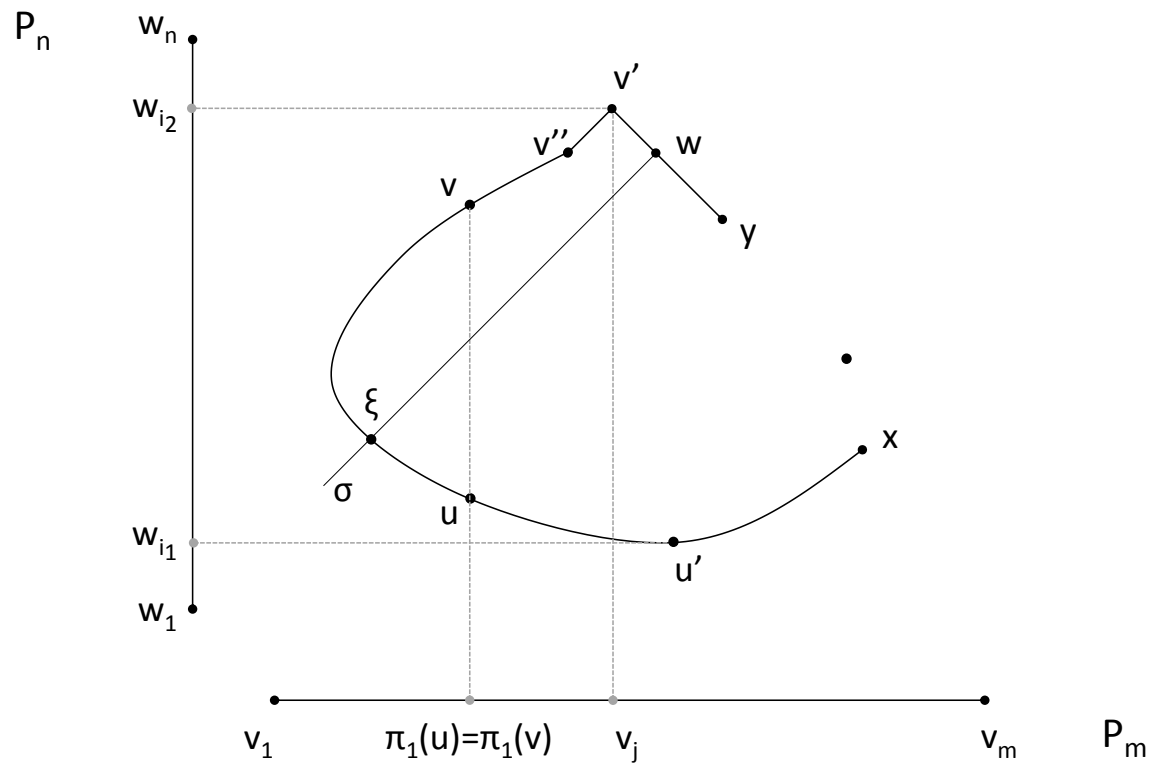

Figure 4. For any geodesic $\gamma$ in $P_{m} \times P_{n}$ with $\pi_{1}(u)=\pi_{1}(v)$ for some different vertices $u, v$ in $\gamma$, then $L(\gamma) \leq n-1$.

Theorem 17. Let $P_{m}, P_{n}$ be two path graphs with $m \geq n \geq 2$. If $n=2$, then $\delta\left(P_{m} \times P_{2}\right)=0$. If $n \geq 3$, then

$$
\min \left\{\frac{m}{2}, n-1\right\}-1 \leq \delta\left(P_{m} \times P_{n}\right) \leq \min \left\{\frac{m}{2}, n\right\}-\frac{1}{2} .
$$

Furthermore, if $m \leq 2 n-3$ and $m$ is odd, then $\delta\left(P_{m} \times P_{n}\right)=(m-1) / 2$.

Proof. If $m \geq 2$, then $P_{m} \times P_{2}$ has two connected components isomorphic to $P_{m}$, and $\delta\left(P_{m} \times P_{2}\right)=0$.

Assume that $n \geq 3$. By symmetry, it suffices to prove the inequalities for $\delta\left(\left(P_{m} \times P_{n}\right)^{1}\right)$. Hence, Theorem 16 and Remark 4 give $\delta\left(\left(P_{m} \times P_{n}\right)^{1}\right) \leq \frac{m-1}{2}$. By Theorem 7 , there exists a geodesic triangle $T=\{x, y, z\} \in \mathbb{T}_{1}\left(P_{m} \times P_{n}\right)$ with $p \in \gamma_{1}:=[x y], \gamma_{2}:=[x z], \gamma_{3}:=[y z]$, and $\delta\left(\left(P_{m} \times P_{n}\right)^{1}\right)=\delta(T)=$ $d_{\left(P_{m} \times P_{n}\right)^{1}}\left(p, \gamma_{2} \cup \gamma_{3}\right)$. Let $u \in V\left(\gamma_{1}\right)$ such that $d_{\left(P_{m} \times P_{n}\right)^{1}}(p, u) \leq 1 / 2$.

To prove $\delta\left(\left(P_{m} \times P_{n}\right)^{1}\right) \leq n-1 / 2$, we consider two cases.

Assume first that there is at least a vertex $v \in V\left(\left(P_{m} \times P_{n}\right)^{1}\right) \cap T \backslash\{u\}$ such that $\pi_{1}(u)=\pi_{1}(v)$. If $v \notin \gamma_{1}$, then $v \in \gamma_{2} \cup \gamma_{3}$ and

$$
\delta(T)=d_{\left(P_{m} \times P_{n}\right)^{1}}\left(p, \gamma_{2} \cup \gamma_{3}\right) \leq 1 / 2+d_{\left(P_{m} \times P_{n}\right)^{1}}(u, v) \leq n-1 / 2 .
$$

If $v \in \gamma_{1}$, then $L\left(\gamma_{1}\right) \leq n-1$ by Lemma 9 , and

$$
\delta(T)=d_{\left(P_{m} \times P_{n}\right)^{1}}\left(p, \gamma_{2} \cup \gamma_{3}\right) \leq d_{\left(P_{m} \times P_{n}\right)^{1}}(p,\{x, y\}) \leq(n-1) / 2<n-1 / 2 .
$$

Assume now that there is not a vertex $v \in V\left(\left(P_{m} \times P_{n}\right)^{1}\right) \cap T \backslash\{u\}$ such that $\pi_{1}(u)=\pi_{1}(v)$. Then, there exist two different vertices $v_{1}, v_{2}$ in $T \backslash\{u\}$ such that $d_{\left(P_{m} \times P_{n}\right)^{1}}\left(u, v_{1}\right)=d_{\left(P_{m} \times P_{n}\right)^{1}}\left(u, v_{2}\right)=1$, and $\pi_{1}\left(v_{1}\right)=\pi_{1}\left(v_{2}\right)$. If $v_{1}$ or $v_{2}$ belongs to $\gamma_{2} \cup \gamma_{3}$, then $\delta(T)=d_{\left(P_{m} \times P_{n}\right)^{1}}\left(p, \gamma_{2} \cup \gamma_{3}\right) \leq 3 / 2 \leq n-1 / 2$. Otherwise, $v_{1}, v_{2} \in \gamma_{1} \backslash\{u\}$. Lemma 9 gives $L\left(\gamma_{1}\right) \leq n-1$, and we have that

$$
\delta(T)=d_{\left(P_{m} \times P_{n}\right)^{1}}\left(p, \gamma_{2} \cup \gamma_{3}\right) \leq d_{\left(P_{m} \times P_{n}\right)^{1}}(p,\{x, y\}) \leq(n-1) / 2<n-1 / 2 .
$$


To prove the lower bound, denote the vertices of $P_{m}$ and $P_{n}$ by $V\left(P_{m}\right)=\left\{w_{1}, w_{2}, w_{3}, \ldots, w_{m}\right\}$ and $V\left(P_{n}\right)=\left\{v_{1}, v_{2}, v_{3}, \ldots, v_{n}\right\}$, with $\left[w_{i}, w_{i+1}\right] \in E\left(P_{m}\right)$ for $1 \leq i<m$ and $\left[v_{i}, v_{i+1}\right] \in E\left(P_{n}\right)$ for $1 \leq i<n$.

Let $\left(P_{m} \times P_{n}\right)^{1}$ be the connected component of $P_{m} \times P_{n}$ containing $\left(w_{1}, v_{n-1}\right)$.

Assume first that $m \geq 2 n-3$. Consider the following curves in $\left(P_{m} \times P_{n}\right)^{1}$ :

$$
\begin{aligned}
\gamma_{1} & :=\left[\left(w_{1}, v_{n-1}\right),\left(w_{2}, v_{n}\right)\right] \cup\left[\left(w_{2}, v_{n}\right),\left(w_{3}, v_{n-1}\right)\right] \cup\left[\left(w_{3}, v_{n-1}\right),\left(w_{4}, v_{n}\right)\right] \cup \cdots \cup\left[\left(w_{2 n-4}, v_{n}\right),\left(w_{2 n-3}, v_{n-1}\right)\right], \\
\gamma_{2} & :=\left[\left(w_{1}, v_{n-1}\right),\left(w_{2}, v_{n-2}\right)\right] \cup\left[\left(w_{2}, v_{n-2}\right),\left(w_{3}, v_{n-3}\right)\right] \cup \cdots \cup\left[\left(w_{n-2}, v_{2}\right),\left(w_{n-1}, v_{1}\right)\right] \cup\left[\left(w_{n-1}, v_{1}\right),\left(w_{n}, v_{2}\right)\right] \\
& \cup \cdots \cup\left[\left(w_{2 n-4}, v_{n-2}\right),\left(w_{2 n-3}, v_{n-1}\right)\right] .
\end{aligned}
$$

Corollary 1 gives that $\gamma_{1}, \gamma_{2}$ are geodesics. If $B$ is the geodesic bigon $B=\left\{\gamma_{1}, \gamma_{2}\right\}$, then Remark 4 gives that

$$
\delta\left(P_{m} \times P_{n}\right) \geq \delta(B) \geq d_{\left(P_{m} \times P_{n}\right)^{1}}\left(\left(w_{n-1}, v_{1}\right), \gamma_{1}\right)=n-2 .
$$

If $m$ is odd with $m \leq 2 n-3$, then $n-(m+1) / 2 \geq 1$ and we can consider the curves in $\left(P_{m} \times P_{n}\right)^{1}$ :

$$
\begin{aligned}
\gamma_{1} & :=\left[\left(w_{1}, v_{n-1}\right),\left(w_{2}, v_{n}\right)\right] \cup\left[\left(w_{2}, v_{n}\right),\left(w_{3}, v_{n-1}\right)\right] \cup\left[\left(w_{3}, v_{n-1}\right),\left(w_{4}, v_{n}\right)\right] \cup \cdots \cup\left[\left(w_{m-1}, v_{n}\right),\left(w_{m}, v_{n-1}\right)\right], \\
\gamma_{2} & :=\left[\left(w_{1}, v_{n-1}\right),\left(w_{2}, v_{n-2}\right)\right] \cup\left[\left(w_{2}, v_{n-2}\right),\left(w_{3}, v_{n-3}\right)\right] \cup \cdots \cup\left[\left(w_{(m+1) / 2-1}, v_{n-(m+1) / 2+1}\right),\left(w_{(m+1) / 2}, v_{n-(m+1) / 2}\right)\right] \\
& \cup\left[\left(w_{(m+1) / 2}, v_{n-(m+1) / 2}\right),\left(w_{(m+1) / 2+1}, v_{n-(m+1) / 2+1}\right)\right] \cup \cdots \cup\left[\left(w_{m-1}, v_{n-2}\right),\left(w_{m}, v_{n-1}\right)\right] .
\end{aligned}
$$

Corollary 1 gives that $\gamma_{1}, \gamma_{2}$ are geodesics. If $B=\left\{\gamma_{1}, \gamma_{2}\right\}$, then Remark 4 gives that

$$
\delta\left(P_{m} \times P_{n}\right) \geq \delta(B) \geq d_{\left(P_{m} \times P_{n}\right)^{1}}\left(\left(w_{(m+1) / 2}, v_{n-(m+1) / 2}\right), \gamma_{1}\right)=(m-1) / 2 .
$$

By Remark 4, if $m$ is even with $m-1 \leq 2 n-3$, then we have that

$$
\delta\left(P_{m} \times P_{n}\right) \geq \delta\left(P_{m-1} \times P_{n}\right) \geq(m-2) / 2 .
$$

Hence,

$$
\delta\left(P_{m} \times P_{n}\right) \geq\left\{\begin{array}{ll}
n-2, & \text { if } m \geq 2 n-3 \\
(m-2) / 2, & \text { if } m \leq 2 n-2
\end{array}\right\}=\min \left\{n-2, \frac{m-2}{2}\right\}=\min \left\{\frac{m}{2}, n-1\right\}-1
$$

Furthermore, if $m \leq 2 n-3$ and $m$ is odd, then we have proven $(m-1) / 2 \leq \delta\left(P_{m} \times P_{n}\right) \leq$ $(m-1) / 2$.

Theorem 18. If $G_{1}$ and $G_{2}$ are bipartite connected graphs with $k_{1}:=\operatorname{diam} V\left(G_{1}\right)$ and $k_{2}:=\operatorname{diam} V\left(G_{2}\right)$ such that $k_{1} \geq k_{2} \geq 1$, then

$$
\max \left\{\min \left\{\frac{k_{1}-1}{2}, k_{2}-1\right\}, \delta\left(G_{1}\right), \delta\left(G_{2}\right)\right\} \leq \delta\left(G_{1} \times G_{2}\right) \leq \frac{k_{1}}{2} .
$$

Furthermore, if $k_{1} \leq 2 k_{2}-2$ and $k_{1}$ is even, then $\delta\left(G_{1} \times G_{2}\right)=k_{1} / 2$.

Proof. Corollary 1, Theorem 16 and Remark 3 give us the upper bound.

To prove the lower bound, we can see that there exist two path graphs $P_{k_{1}+1}, P_{k_{2}+1}$ which are isometric subgraphs of $G_{1}$ and $G_{2}$, respectively. It is easy to check that $P_{k_{1}+1} \times P_{k_{2}+1}$ is an isometric subgraph of $G_{1} \times G_{2}$. By Lemma 3 and Theorem 17, we have

$$
\min \left\{\frac{k_{1}-1}{2}, k_{2}-1\right\} \leq \delta\left(P_{k_{1}+1} \times P_{k_{2}+1}\right) \leq \delta\left(G_{1} \times G_{2}\right) .
$$

Using a similar argument as above, we have $\delta\left(P_{2} \times G_{2}\right) \leq \delta\left(G_{1} \times G_{2}\right)$ and $\delta\left(G_{1} \times P_{2}\right) \leq \delta\left(G_{1} \times G_{2}\right)$. Thus, since $\left(G_{1} \times P_{2}\right)^{i} \simeq G_{1}$ and $\left(P_{2} \times G_{2}\right)^{i} \simeq G_{2}$ for $i \in\{1,2\}$, we obtain the first statement. 
Furthermore, if $k_{1}+1 \leq 2\left(k_{2}+1\right)-3$ and $k_{1}+1$ is odd, then Theorem 17 gives $\delta\left(P_{k_{1}+1} \times P_{k_{2}+1}\right)=$ $k_{1} / 2$, and we conclude $\delta\left(G_{1} \times G_{2}\right)=k_{1} / 2$.

The following result deals just with odd cycles since otherwise we can apply Theorem 18.

Theorem 19. For every odd number $m \geq 3$ and every $n \geq 2$,

$$
\delta\left(C_{m} \times P_{n}\right)= \begin{cases}m / 2, & \text { if } n-1 \leq m, \\ (n-1) / 2, & \text { if } m<n-1<2 m, \\ m-1 / 2, & \text { if } n-1 \geq 2 m .\end{cases}
$$

Proof. Let $V\left(C_{m}\right)=\left\{w_{1}, \ldots, w_{m}\right\}$ and $V\left(P_{n}\right)=\left\{v_{1}, \ldots, v_{n}\right\}$ be the sets of vertices in $C_{m}$ and $P_{n}$, respectively, such that $\left[w_{1}, w_{m}\right],\left[w_{j}, w_{j+1}\right] \in E\left(C_{m}\right)$ and $\left[v_{i}, v_{i+1}\right] \in E\left(P_{n}\right)$ for $j \in\{1, \ldots, m-1\}$, $i \in\{1, \ldots, n-1\}$. Note that for $1 \leq j, r \leq m$ and $1 \leq i, s \leq n$, we have $d_{C_{m} \times P_{n}}\left(\left(w_{j}, v_{i}\right),\left(w_{r}, v_{s}\right)\right)=$ $\max \{|i-s|,|j-r|\}$, if $|i-s| \equiv|j-r|(\bmod 2), \operatorname{or} d_{C_{m} \times P_{n}}\left(\left(w_{j}, v_{i}\right),\left(w_{r}, v_{s}\right)\right)=\max \{|i-s|, m-|j-r|\}$, if $|i-s| \not \equiv|j-r|(\bmod 2)$. Besides, we have $\operatorname{diam}\left(C_{m} \times P_{n}\right)=\operatorname{diam}\left(V\left(C_{m} \times P_{n}\right)\right)$, i.e., $\operatorname{diam}\left(C_{m} \times\right.$ $\left.P_{n}\right)=m$ if $n-1 \leq m$, and $\operatorname{diam}\left(C_{m} \times P_{n}\right)=n-1$ if $n-1>m$. Thus, by Theorem 16 , we have

$$
\delta\left(C_{m} \times P_{n}\right) \leq \begin{cases}m / 2, & \text { if } n-1 \leq m, \\ (n-1) / 2, & \text { if } n-1>m .\end{cases}
$$

Assume first that $n-1 \leq m$. Note that $C_{m} \times P_{2} \simeq C_{2 m}$ and $C_{m} \times P_{n^{\prime}}$ is an isometric subgraph of $C_{m} \times P_{n}$, if $n^{\prime} \leq n$. By Lemma 3, we have $\delta\left(C_{m} \times P_{n}\right) \geq \delta\left(C_{2 m}\right)=m / 2$, and we obtain the result in this case.

Assume now that $n-1>m$. Consider the geodesic triangle $T$ in $C_{m} \times P_{n}$ defined by the following geodesics

$$
\begin{aligned}
\gamma_{1}:= & {\left[\left(w_{1}, v_{n}\right),\left(w_{2}, v_{n-1}\right)\right] \cup\left[\left(w_{2}, v_{n-1}\right),\left(w_{3}, v_{n}\right)\right] \cup\left[\left(w_{3}, v_{n}\right),\left(w_{4}, v_{n-1}\right)\right] \cup \ldots \cup\left[\left(w_{m-1}, v_{n-1}\right),\left(w_{m}, v_{n}\right)\right], } \\
\gamma_{2}:= & {\left[\left(w_{(m+1) / 2}, v_{1}\right),\left(w_{(m-1) / 2}, v_{2}\right)\right] \cup\left[\left(w_{(m-1) / 2}, v_{2}\right),\left(w_{(m-3) / 2}, v_{3}\right)\right] \cup \ldots \cup\left[\left(w_{2}, v_{(m-1) / 2}\right),\left(w_{1}, v_{(m+1) / 2}\right)\right] \cup } \\
& {\left[\left(w_{1}, v_{(m+1) / 2}\right),\left(w_{m}, v_{(m+3) / 2}\right)\right] \cup\left[\left(w_{m}, v_{(m+3) / 2}\right),\left(w_{1}, v_{(m+5) / 2}\right)\right] \cup\left[\left(w_{1}, v_{(m+5) / 2}\right),\left(w_{m}, v_{(m+7) / 2}\right)\right] \cup \ldots, } \\
\gamma_{3}:= & {\left[\left(w_{(m+1) / 2}, v_{1}\right),\left(w_{(m+3) / 2}, v_{2}\right)\right] \cup\left[\left(w_{(m+3) / 2}, v_{2}\right),\left(w_{(m+5) / 2}, v_{3}\right)\right] \cup \ldots \cup\left[\left(w_{m-1}, v_{(m-1) / 2}\right),\left(w_{m}, v_{(m+1) / 2}\right)\right] \cup } \\
& {\left[\left(w_{m}, v_{(m+1) / 2}\right),\left(w_{1}, v_{(m+3) / 2}\right)\right] \cup\left[\left(w_{1}, v_{(m+3) / 2}\right),\left(w_{m}, v_{(m+5) / 2}\right)\right] \cup\left[\left(w_{m}, v_{(m+5) / 2}\right),\left(w_{1}, v_{(m+7) / 2}\right)\right] \cup \ldots, }
\end{aligned}
$$

where $\left(w_{1}, v_{n}\right)$ (respectively, $\left.\left(w_{m}, v_{n}\right)\right)$ is an endpoint of either $\gamma_{2}$ or $\gamma_{3}$, depending of the parity of $n$. Since $T$ is a geodesic triangle in $C_{m} \times P_{n}$, we have $\delta\left(C_{m} \times P_{n}\right) \geq \delta(T)$. If $n-1<2 m$ and $M$ is the midpoint of the geodesic $\gamma_{3}$, then $\delta\left(C_{m} \times P_{n}\right) \geq \delta(T)=d_{C_{m} \times P_{n}}\left(M, \gamma_{1} \cup \gamma_{2}\right)=L\left(\gamma_{3}\right) / 2=(n-1) / 2$. Therefore, the result for $m<n-1<2 m$ follows.

Finally, assume that $n-1 \geq 2 m$. Let us consider $N \in \gamma_{3}$ such that $d_{C_{m} \times P_{n}}\left(N,\left(w_{(m+1) / 2}, v_{1}\right)\right)=$ $m-1 / 2$. Thus, $\delta\left(C_{m} \times P_{n}\right) \geq \delta(T) \geq d_{C_{m} \times P_{n}}\left(N, \gamma_{1} \cup \gamma_{2}\right)=d_{C_{m} \times P_{n}}\left(N,\left(w_{(m+1) / 2}, v_{1}\right)\right)=m-1 / 2$. To finish the proof, it suffices to prove that $\delta\left(C_{m} \times P_{n}\right) \leq m-1 / 2$. Seeking for a contradiction, assume that $\delta\left(C_{m} \times P_{n}\right)>m-1 / 2$. By Theorems 6 and 7 , there is a geodesic triangle $\triangle=\{x, y, z\} \in$ $\mathbb{T}_{1}\left(C_{m} \times P_{n}\right)$ and $p \in[x y]$ with $d_{C_{m} \times P_{n}}(p,[y z] \cup[z x])=\delta\left(C_{m} \times P_{n}\right) \geq m-1 / 4$. Then, $L([x y])=$ $d_{C_{m} \times P_{n}}(x, p)+d_{C_{m} \times P_{n}}(p, y) \geq 2 m-1 / 2$. Let $V_{x}$ (respectively, $V_{y}$ ) be the closest vertex to $x$ (respectively, $y)$ in $[x y]$, and consider a vertex $V_{p}$ in $[x y]$ such that $d_{C_{m} \times P_{n}}\left(p, V\left(C_{m} \times P_{n}\right)\right)=d_{C_{m} \times P_{n}}\left(p, V_{p}\right)$. Note that $d_{C_{m} \times P_{n}}(p,[y z] \cup[z x]) \geq m-1 / 4$ implies that $d_{C_{m} \times P_{n}}\left(p, V_{p}\right) \leq 1 / 2$. Since $x, y, z \in J\left(C_{m} \times P_{n}\right)$, we have $d_{C_{m} \times P_{n}}\left(V_{x}, V_{y}\right) \geq 2 m-1>m$ and, consequently, $\pi_{2}([x y])$ is a geodesic in $P_{n}$. Since $\pi_{2}([y z] \cup[z x])$ is a path in $P_{n}$ joining $\pi_{2}(x)$ and $\pi_{2}(y)$, there exists a vertex $(u, v) \in[x z] \cup[z y]$ such that $\pi_{2}\left(V_{p}\right)=v$ and $u \neq \pi_{1}\left(V_{p}\right)$. Therefore, $d_{C_{m} \times P_{n}}\left(V_{p},(u, v)\right) \leq m-1$ and, consequently, $d_{C_{m} \times P_{n}}(p,[x z] \cup[z y]) \leq$ $d_{C_{m} \times P_{n}}\left(p, V_{p}\right)+d_{C_{m} \times P_{n}}\left(V_{p},[x z] \cup[z y]\right) \leq 1 / 2+m-1$, leading to contradiction. 


\section{Conclusions}

In this paper, we characterize in many cases the hyperbolic direct product of graphs. Here, the situation is more complex than with other graph products, partly because the direct product of two bipartite graphs is already disconnected and the formula for the distance in $G_{1} \times G_{2}$ is more complicated than in the case of other products of graphs. Although in the study of hyperbolicity in a general context the hypothesis on the existence (or non-existence) of odd cycles is artificial, in the study of hyperbolic direct products, it is an essential hypothesis. We have proven that, if $G_{1} \times G_{2}$ is hyperbolic, then one factor is hyperbolic and the other one is bounded. Besides, we prove that this necessary condition is also sufficient in many cases. If $G_{1}$ is a hyperbolic graph and $G_{2}$ is a bounded graph, then we prove that $G_{1} \times G_{2}$ is hyperbolic when $G_{2}$ has some odd cycle or $G_{1}$ and $G_{2}$ do not have odd cycles. Otherwise, the characterization of hyperbolic direct products is a more difficult task. If $G_{1}$ has some odd cycle and $G_{2}$ does not have odd cycles, we provide sufficient conditions for non-hyperbolicity and hyperbolicity, respectively. Besides, we characterize the hyperbolicity of $G_{1} \times G_{2}$ under some additional conditions.

A natural open problem is the complete characterization of hyperbolic direct products.

A second open problem is to compute the precise value of the hyperbolicity constant of the graphs appearing in Theorems 17 and 18 with unknown hyperbolicity constant.

Direct product of graphs is a subject closely related to lift of graphs, which have been intensively studied (see, e.g., [82] and the references therein). Another interesting problem is to study the hyperbolicity of lift of graphs. We think that it is possible to obtain some similar results in this context, although the odd cycles may not play an important role in the study of hyperbolic lifts of graphs.

Author Contributions: The authors contributed equally to this work.

Funding: This work was supported in part by four grants from Ministerio de Economía y Competititvidad (MTM2012-30719, MTM2013-46374-P, MTM2016-78227-C2-1-P and MTM2015-69323-REDT), Spain.

Acknowledgments: We thank the referees for their suggestions and helpful remarks.

Conflicts of Interest: The authors declare no conflict of interest. The founding sponsors had no role in the design of the study; in the collection, analyses, or interpretation of data; in the writing of the manuscript, and in the decision to publish the results.

\section{References}

1. Hammack, R.; Imrich, W.; Klavžar, S. Handbook of Product Graphs, 2rd ed.; Discrete Mathematics and Its Applications Series; CRC Press: Boca Raton, FL, USA, 2011.

2. Weichsel, P.M. The Kronecker product of graphs. Proc. Am. Math. Soc. 1962, 13, 47-52. [CrossRef]

3. Bendall, S.; Hammack, R. Centers of $n$-fold tensor products of graphs. Discuss. Math. Graph Theory 2004, 24, 491-501. [CrossRef]

4. Brešar, B.; Imrich, W.; Klavžar, S.; Zmazek, B. Hypercubes as direct products. SIAM J. Discret. Math. 2005, 18, 778-786. [CrossRef]

5. Hammack, R. Minimum cycle bases of direct products of bipartite graphs. Australas. J. Combin. 2006, 36, 213-222.

6. Imrich, W.; Rall, D.F. Finite and infinite hypercubes as direct products. Australas. J. Combin. 2006, 36, 83-90.

7. Imrich, W.; Stadler, P. A prime factor theorem for a generalized direct product. Discuss. Math. Graph Theory 2006, 26, 135-140. [CrossRef]

8. Jha, P.K.; Klavžar, S. Independence in direct-product graphs. Ars Combin. 1998, 50, 53-63.

9. Balakrishnan, R.; Paulraja, P. Hamilton cycles in tensor product of graphs. Discret. Math. 1998, 186, 1-13. [CrossRef]

10. Kheddouci, H.; Kouider, M. Hamiltonian cycle decomposition of Kronecker product of some cubic graphs by cycles. J. Combin. Math. Combin. Comput. 2000, 32, 3-22. 
11. Imrich, W.; Klavžar, S. Product Graphs: Structure and Recognition; John Wiley \& Sons: New York, NY, USA, 2000.

12. Zhu, X. A survey on Hedetniemi's conjecture. Taiwanese J. Math. 1998, 2, 1-24. [CrossRef]

13. Imrich, W. Factoring cardinal product graphs in polynomial time. Discr. Math. 1998, 192, 119-144. [CrossRef]

14. Alonso, J.; Brady, T.; Cooper, D.; Delzant, T.; Ferlini, V.; Lustig, M.; Mihalik, M.; Shapiro, M.; Short, H. Notes on word hyperbolic groups. In Group Theory from a Geometrical Viewpoint; Ghys, E., Haefliger, A., Verjovsky, A., Eds.; World Scientific: Singapore, 1992.

15. Ghys, E.; de la Harpe, P. Sur les Groupes Hyperboliques d'après Mikhael Gromov; Progress in Mathematics 83; Birkhäuser Boston Inc.: Boston, MA, USA, 1990.

16. Gromov, M. Hyperbolic groups. In Essays in Group Theory; Gersten, S.M., Ed.; Mathematical Sciences Research Institute Publications; Springer: Berlin, Germany, 1987; Volume 8, pp. 75-263.

17. Oshika, K. Discrete Groups; AMS Bookstore: Providence, RI, USA, 2002.

18. Charney, R. Artin groups of finite type are biautomatic. Math. Ann. 1992, 292, 671-683. [CrossRef]

19. Chepoi, V.; Estellon, B. Packing and covering $\delta$-hyperbolic spaces by balls. In Approximation, Randomization, and Combinatorial Optimization. Algorithms and Techniques; Springer: Berlin/Heidelberg, Germany, 2007; pp. 59-73.

20. Eppstein, D. Squarepants in a tree: Sum of subtree clustering and hyperbolic pants decomposition. In Proceedings of the eighteenth annual ACM-SIAM symposium on Discrete algorithms (SODA'2007), Barcelona, Spain, 16-19 January 2017; pp. 29-38.

21. Gavoille, C.; Ly, O. Distance labeling in hyperbolic graphs. In Proceedings of the International Symposium on Algorithms and Computation (ISAAC); Springer: Berlin/Heidelberg, Germany, 2005; pp. 171-179.

22. Krauthgamer, R.; Lee, J.R. Algorithms on negatively curved spaces. In Proceedings of the 47th Annual IEEE Symposium on Foundations of Computer Science (FOCS’06), Berkeley, CA, USA, 21-24 October 2006; pp. 1-11.

23. Shavitt, Y.; Tankel, T. On Internet embedding in hyperbolic spaces for overlay construction and distance estimation. IEEE/ACM Trans. Netw. 2008, 16, 25-36. [CrossRef]

24. Verbeek, K.; Suri, S. Metric embeddings, hyperbolic space and social networks. In Proceedings of the 30th Annual Symposium on Computational Geometry, Kyoto, Japan, 8-11 June 2014; pp. 501-510.

25. Wu, Y.; Zhang, C. Chordality and hyperbolicity of a graph. Electr. J. Comb. 2011, 18, P43.

26. Abu-Ata, M.; Dragan, F.F. Metric tree-like structures in real-life networks: An empirical study. Networks 2016, 67, 49-68. [CrossRef]

27. Adcock, A.B.; Sullivan, B.D.; Mahoney, M.W. Tree-like structure in large social and information networks. In Proceedings of the 13th Int Conference Data Mining (ICDM), Dallas, TX, USA, 7-10 December 2013; pp. 1-10.

28. Krioukov, D.; Papadopoulos, F.; Kitsak, M.; Vahdat, A.; Boguná, M. Hyperbolic geometry of complex networks. Phys. Rev. E 2010, 82, 036106. [CrossRef] [PubMed]

29. Montgolfier, F.; Soto, M.; Viennot, L. Treewidth and Hyperbolicity of the Internet. In Proceedings of the 10th IEEE International Symposium on Network Computing and Applications (NCA), Cambridge, MA, USA, 25-27 August 2011; pp. 25-32.

30. Chepoi, V.; Dragan, F.F.; Vaxès, Y. Core congestion is inherent in hyperbolic networks. In Proceedings of the Twenty-Eighth Annual ACM-SIAM Symposium on Discrete Algorithms, Barcelona, Spain, 16-19 January 2017; pp. 2264-2279.

31. Grippo, E.; Jonckheere, E.A. Effective resistance criterion for negative curvature: application to congestion control. In Proceedings of the 2016 IEEE Conference on Control Applications (CCA), Buenos Aires, Argentina, 19-22 September 2016.

32. Jonckheere, E.A. Contrôle du traffic sur les réseaux à géométrie hyperbolique-Vers une théorie géométrique de la sécurité l'acheminement de l'information. J. Eur. Syst. Autom. 2002, 8, 45-60.

33. Jonckheere, E.A.; Lohsoonthorn, P. Geometry of network security. In Proceedings of the 2004 American Control Conference, Boston, MA, USA, 30 June-2 July 2004; pp. 111-151.

34. Li, S.; Tucci, G.H. Traffic Congestion in Expanders, $(p, \delta)$-Hyperbolic Spaces and Product of Trees. Int. Math. 2015, 11, 134-142.

35. Shang, Y. Lack of Gromov-hyperbolicity in colored random networks. Pan-Am. Math. J. 2011, 21, 27-36. 
36. Shang, Y. Lack of Gromov-hyperbolicity in small-world networks. Cent. Eur. J. Math. 2012, 10, 1152-1158. [CrossRef]

37. Shang, Y. Non-hyperbolicity of random graphs with given expected degrees. Stoch. Models 2013, 29, 451-462. [CrossRef]

38. Gosak, M.; Markovič, R.; Dolenšek, J.; Slak Rupnik, M.; Marhl, M.; Stožer, A.; Perc, M. Network science of biological systems at different scales: A review. Phys. Life Rev. 2018, 24, 118-135. [CrossRef] [PubMed]

39. Tourís, E. Graphs and Gromov hyperbolicity of non-constant negatively curved surfaces. J. Math. Anal. Appl. 2011, 380, 865-881. [CrossRef]

40. Bermudo, S.; Rodríguez, J.M.; Sigarreta, J.M. Computing the hyperbolicity constant. Comput. Math. Appl. 2011, 62, 4592-4595. [CrossRef]

41. Bermudo, S.; Rodríguez, J.M.; Sigarreta, J.M.; Vilaire, J.-M. Gromov hyperbolic graphs. Discr. Math. 2013, 313, 1575-1585. [CrossRef]

42. Brinkmann, G.; Koolen J.; Moulton, V. On the hyperbolicity of chordal graphs. Ann. Comb. 2001, 5, 61-69. [CrossRef]

43. Chepoi, V.; Dragan, F.F.; Estellon, B.; Habib, M.; Vaxes, Y. Notes on diameters, centers, and approximating trees of $\delta$-hyperbolic geodesic spaces and graphs. Electr. Notes Discr. Math. 2008, 31, 231-234. [CrossRef]

44. Dragan, F.; Mohammed, A. Slimness of graphs. arXiv 2017, arXiv:1705.09797.

45. Martínez-Pérez, A. Chordality properties and hyperbolicity on graphs. Electr. J. Comb. 2016, 23, P3.51.

46. Michel, J.; Rodríguez, J.M.; Sigarreta, J.M.; Villeta, M. Gromov hyperbolicity in Cartesian product graphs. Proc. Indian Acad. Sci. Math. Sci. 2010, 120, 1-17. [CrossRef]

47. Rodríguez, J.M.; Sigarreta, J.M.; Vilaire, J.-M.; Villeta, M. On the hyperbolicity constant in graphs. Discr. Math. 2011, 311, 211-219. [CrossRef]

48. Chen, B.; Yau, S.-T.; Yeh, Y.-N. Graph homotopy and Graham homotopy. Discret. Math. 2001, 241, $153-170$. [CrossRef]

49. Shang, Y. On the likelihood of forests. Phys. A Stat. Mech. Appl. 2016, 456, 157-166. [CrossRef]

50. Fournier, H.; Ismail, A.; Vigneron, A. Computing the Gromov hyperbolicity of a discrete metric space. Inf. Process. Lett. 2015, 115, 576-579. [CrossRef]

51. Papasoglu, P. An algorithm detecting hyperbolicity. In Geometric and Computational Perspectives on Infinite Groups, DIMACS; Series in Discrete Mathematics and Theoretical Computer Science; AMS: Hong Kong, China, 1996; Volume 25, pp.193-200.

52. Chalopin, J.; Chepoi, V.; Papasoglu, P.; Pecatte, T. Cop and robber game and hyperbolicity. SIAM J. Discr. Math. 2015, 28, 1987-2007. [CrossRef]

53. Sigarreta, J.M. Hyperbolicity in median graphs. Proc. Indian Acad. Sci. Math. Sci. 2013, 123, $455-467$. [CrossRef]

54. Carballosa, W.; Rodríguez, J.M.; Sigarreta, J.M. New inequalities on the hyperbolicity constant of line graphs. Ars Combin. 2016, 129, 367-386.

55. Carballosa, W.; Rodríguez, J.M.; Sigarreta, J.M.; Villeta, M. Gromov hyperbolicity of line graphs. Electr. J. Comb. 2011, 18, P210.

56. Cohen, N.; Coudert, D.; Ducoffe, G.; Lancin, A. Applying clique-decomposition for computing Gromov hyperbolicity. Theor. Comp. Sci. 2017./j.tcs.2017.06.001. [CrossRef]

57. Pestana, D.; Rodríguez, J.M.; Sigarreta, J.M.; Villeta, M. Gromov hyperbolic cubic graphs. Central Eur. J. Math. 2012, 10, 1141-1151. [CrossRef]

58. Bermudo, S.; Rodríguez, J.M.; Sigarreta, J.M.; Tourís, E. Hyperbolicity and complement of graphs. Appl. Math. Lett. 2011, 24, 1882-1887. [CrossRef]

59. Hernández, J.C.; Rodríguez, J.M.; Sigarreta, J.M.; Torres-Nuñez, Y.; Villeta, M. Gromov hyperbolicity of regular graphs. Ars Combin. 2017, 130, 395-416.

60. Bermudo, S.; Carballosa, W.; Rodríguez, J.M.; Sigarreta, J.M. On the hyperbolicity of edge-chordal and path-chordal graphs. Filomat 2016, 30, 2599-2607. [CrossRef]

61. Carballosa, W.; Portilla, A.; Rodríguez, J.M.; Sigarreta, J.M. Planarity and hyperbolicity in graphs. Graphs Combin. 2015, 31, 1311-1324. [CrossRef]

62. Portilla, A.; Rodríguez, J.M.; Sigarreta, J.M.; Vilaire, J.-M. Gromov hyperbolic tessellation graphs. Utilitas Math. 2015, 97, 193-212. 
63. Coudert, D.; Ducoffe, G. On the hyperbolicity of bipartite graphs and intersection graphs. Discr. Appl. Math. 2016, 214, 187-195. [CrossRef]

64. Calegari, D.; Fujiwara, K. Counting subgraphs in hyperbolic graphs with symmetry. J. Math. Soc. Jpn. 2015, 67, 1213-1226. [CrossRef]

65. Cantón, A.; Granados, A.; Pestana, D.; Rodríguez, J.M. Gromov hyperbolicity of periodic graphs. Bull. Malays. Math. Sci. Soc. 2016, 39, S89-S116. [CrossRef]

66. Cantón, A.; Granados, A.; Pestana, D.; Rodríguez, J.M. Gromov hyperbolicity of periodic planar graphs. Acta Math. Sin. 2014, 30, 79-90. [CrossRef]

67. Koolen, J.H.; Moulton, V. Hyperbolic Bridged Graphs. Eur. J. Comb. 2002, 23, 683-699. [CrossRef]

68. Rodríguez, J.M. Characterization of Gromov hyperbolic short graphs. Acta Math. Sin. 2014, 30, 197-212. [CrossRef]

69. Carballosa, W.; Rodríguez, J.M.; Rosario, O.; Sigarreta, J.M. Gromov hyperbolicity of graph minors. Bull. Iran. Math. Soc. 2018, 44, 481-503. [CrossRef]

70. Bermudo, S.; Rodríguez, J.M.; Rosario, O.; Sigarreta, J.M. Small values of the hyperbolicity constant in graphs. Discr. Math. 2016, 339, 3073-3084. [CrossRef]

71. Granados, A.; Pestana, D.; Portilla, A.; Rodríguez, J.M. Gromov hyperbolicity in Mycielskian Graphs. Symmetry 2017, 9, 131. [CrossRef]

72. Hernández, J.C.; Reyes, R.; Rodríguez, J.M.; Sigarreta, J.M. Mathematical properties on the hyperbolicity of interval graphs. Symmetry 2017, 9, 255. [CrossRef]

73. Carballosa, W.; de la Cruz, A.; Rodríguez, J.M. Gromov hyperbolicity in the Cartesian sum of graphs. Bull. Iran. Math. Soc. 2017, 1-20. [CrossRef]

74. Carballosa, W.; Casablanca, R.M.; de la Cruz, A.; Rodríguez, J.M. Gromov hyperbolicity in strong product graphs. Electr. J. Comb. 2013, 20, 2.

75. Carballosa, W.; de la Cruz, A.; Rodríguez, J.M. Gromov hyperbolicity in lexicographic product graphs. arXiv 2015, arXiv 1506.06034

76. Carballosa, W.; Rodríguez, J.M.; Sigarreta, J.M. Hyperbolicity in the corona and join of graphs. Aequ. Math. 2015, 89, 1311-1327. [CrossRef]

77. Bowditch, B.H. Notes on Gromov's hyperbolicity criterion for path-metric spaces. In Group Theory from a Geometrical Viewpoint; Ghys, E., Haefliger, A., Verjovsky, A., Eds.; World Scientific: River Edge, NJ, USA, 1991; pp. 64-167.

78. Shchur, V. A quantitative version of the Morse lemma and quasi-isometries fixing the ideal boundary. J. Funct. Anal. 2013, 264, 815-836. [CrossRef]

79. Rodríguez, J.M.; Tourís, E. Gromov hyperbolicity through decomposition of metric spaces. Acta Math. Hung. 2004, 103, 53-84. [CrossRef]

80. Alvarez, V.; Portilla, A.; Rodríguez, J.M.; Tourís, E. Gromov hyperbolicity of Denjoy domains. Geom. Dedic. 2006, 121, 221-245. [CrossRef]

81. Bonk, M. Quasi-geodesics segments and Gromov hyperbolic spaces. Geom. Dedicata 1996, 62, $281-298$. [CrossRef]

82. Shang, Y. Random Lifts Of Graphs: Network Robustness Based On The Estrada Index. Appl. Math. E-Notes 2012, 12, 53-61.

(c) 2018 by the authors. Licensee MDPI, Basel, Switzerland. This article is an open access article distributed under the terms and conditions of the Creative Commons Attribution (CC BY) license (http:/ / creativecommons.org/licenses/by/4.0/). 